\title{
Gesundheitsbezogene Lebensqualität nach Schädel-Hirn-Trauma - Einfluss von Selbstwahrnehmung und Krankheitsverarbeitung
}

\author{
Dissertation \\ zur Erlangung des mathematisch-naturwissenschaftlichen Doktorgrades \\ "Doctor rerum naturalium" \\ der Georg-August-Universität Göttingen \\ im Promotionsprogramm Biologie \\ der Georg-August University School of Science (GAUSS) \\ vorgelegt von \\ Nadine Sasse \\ aus Rostock
}

Göttingen, Oktober 2014 


\section{Betreuungsausschuss:}

Prof. Dr. Marcus Hasselhorn, Abteilung Bildung und Entwicklung, Deutsches Institut für Internationale Pädagogische Forschung (DIPF), Frankfurt am Main

Prof. Dr. Nicole von Steinbüchel, Institut für Medizinische Psychologie und Medizinische Soziologie, Universitätsmedizin Göttingen (UMG)

Mitglieder der Prüfungskommission:

Referent: Prof. Dr. Marcus Hasselhorn, Abteilung Bildung und Entwicklung, Deutsches Institut für Internationale Pädagogische Forschung (DIPF), Frankfurt am Main

Korreferentin: Prof. Dr. Nicole von Steinbüchel, Institut für Medizinische Psychologie und Medizinische Soziologie, Universitätsmedizin Göttingen (UMG)

Weitere Mitglieder der Prüfungskommission:

Prof. Dr. Margarete Boos, Abteilung „Sozial- und Kommunikationspsychologie, Georg-Elias-Müller-Institut für Psychologie

Prof. Dr. Uta Lass, Abteilung „Experimentelle Psychologie“, Georg-Elias-Müller-Institut für Psychologie

Prof. Dr. Lars Penke, Abteilung „Biologische Persönlichkeitspsychologie und Diagnostik“, Georg-Elias-Müller-Institut für Psychologie

Prof. Dr. Hannes Rakoczy, Abteilung „Biologische Entwicklungspsychologie“, Georg-Elias-Müller-Institut für Psychologie

Tag der mündlichen Prüfung: 8. Dezember 2014 


\section{DANKSAGUNG}

Herzlich danken möchte ich Herrn Prof. Marcus Hasselhorn für seine ermutigende und geduldige Betreuung und Unterstützung dieses Promotionsvorhabens. Frau Prof. Nicole von Steinbüchel danke ich besonders für die Realisierung dieses Forschungsprojektes, die vielen fachlichen Anregungen und Einblicksmöglichkeiten in damit verbundene Bereiche, ihre Förderung und Unterstützung der wissenschaftlichen Ergebnispräsentation und ihre kritischen Anmerkungen bei der Erstellung und Überarbeitung meiner Arbeiten.

In trauerndem Gedenken möchte ich ebenfalls Frau Carmen Franz von Herzen für ihr maßgeblich unterstützendes Wirken durch Mutzuspruch und schnörkellosem Rat danken. Herrn Dr. Wolfgang Woerner danke ich für seine unermüdliche Unterstützung in der statistischen Bewältigung der umfangreichen Datensätze und Lösung datenbezogener wie auch formaler und inhaltlicher Herausforderungen, sowie Herrn Prof. Henning Gibbons für die fortführende statistische und ermutigende Betreuung und seine kritische Reflexion der Dateninhalte in Hinblick auf den Gesamtkontext dieser Arbeit. Herzlich danken möchte ich auch all meinen Koautoren der dieser Arbeit zugrunde liegenden Publikationen, und hier besonders Prof. Lindsay Wilson und Prof. Klaus von Wild für ihre kritischen Anmerkungen und ihre bereichernde Unterstützung während der Überarbeitung der Manuskripte. Auch möchte ich meinen Kollegen des Instituts für Medizinische Psychologie und Medizinische Soziologie in Göttingen danken für ihre ständige Hilfsbereitschaft und den anregenden wissenschaftlichen Austausch innerhalb unseres Fachbereiches während meiner Promotionszeit.

Meinen besonderen Dank möchte ich gegenüber meinen Eltern zum Ausdruck bringen, die mich immerwährend ermutigend in Rat und Tat unterstützten, in Momenten des Zweifelns aufbauenden Zuspruch spendeten und mir mit ihrem unvermittelten Glauben an mein gesetztes Promotionsziel immer wieder neue Kraft für deren Fortgang und Umsetzung gaben. Meinen engeren Freunden danke ich von Herzen für ihre entgegengebrachte Geduld und Nachsicht, ihre wertvollen Anregungen und auch ihre tatkräftige Unterstützung in herausfordernden Situationen, ebenso wie allen anderen 
Freunden und Bekannten für all die gemeinsamen Unternehmungen und den damit verbundenen angenehmen Stunden mit ihnen, die mir Kraft gebende Freude schenkten.

Und schließlich möchte ich herzlich allen Teilnehmern dieser Studien danken, ohne deren Mitarbeit dieses Promotionsvorhaben nicht möglich gewesen wäre. 


\section{INHALTSVERZEICHNIS}

1. EINLEITUNG

2. FORSCHUNG ZU GESUNDHEITSBEZOGENER LEBENSQUALITÄT, SELBSTWAHRNEHMUNG UND KRANKHEITSVERARBEITUNG NACH SCHÄDEL-HIRN-TRAUMA

2.1 Schädel-Hirn-Trauma 3

$\begin{array}{ll}2.2 \text { Gesundheitsbezogene Lebensqualität } & 7\end{array}$

2.3 Selbstwahrnehmung und gestörte Selbstwahrnehmung 11

$\begin{array}{ll}2.4 \text { Coping und Krankheitsverarbeitung } & 17\end{array}$

3. ZIELSETZUNG UND EIGENE FRAGESTELLUNGEN 22

4. SELBSTWAHRNEHMUNG UND GESUNDHEITSBEZOGENE LEBENSQUALITÄT NACH SCHÄDEL-HIRN-TRAUMA

(Self-awareness and health-related quality of life after traumatic brain injury) 24

$\begin{array}{ll}\text { Abstract } & 25\end{array}$

$\begin{array}{ll}\text { Introduction } & 25\end{array}$

$\begin{array}{ll}\text { Methods } & 26\end{array}$

$\begin{array}{ll}\text { Participants } & 26\end{array}$

$\begin{array}{ll}\text { Measures } & 27\end{array}$

$\begin{array}{ll}\text { Procedure } & 28\end{array}$

$\begin{array}{ll}\text { Statistical analysis } & 28\end{array}$

Results 28

$\begin{array}{ll}\text { Dimensional analyses } & 28\end{array}$

$\begin{array}{ll}\text { Categorical analyses } & 29\end{array}$

$\begin{array}{ll}\text { Exploratory regression analyses } & 29\end{array}$

$\begin{array}{ll}\text { Discussion } & 30\end{array}$

HRQOL and SA $\quad 30$

$\begin{array}{ll}\text { Emotional status and SA } & 31\end{array}$

Sociodemographic and clinical variables and SA 31

$\begin{array}{ll}\text { Severity of injury and SA } & 31\end{array}$

$\begin{array}{ll}\text { Predictors of SA } & 31\end{array}$

$\begin{array}{ll}\text { Study limitations } & 31\end{array}$

$\begin{array}{ll}\text { Conclusion } & 32\end{array}$

$\begin{array}{ll}\text { References } & 32\end{array}$ 
5. KRANKHEITSVERARBEITUNGSSTRATEGIEN VON PERSONEN NACH

SCHÄDEL-HIRN-TRAUMA: ZUSAMMENHÄNGE MIT

GESUNDHEITSBEZOGENER LEBENSQUALITÄT

(Coping strategies in individuals after traumatic brain injury: associations with health-related quality of life) $\quad 34$

$\begin{array}{ll}\text { Abstract } & 35\end{array}$

$\begin{array}{ll}\text { Introduction } & 35\end{array}$

$\begin{array}{ll}\text { Aims } & 36\end{array}$

$\begin{array}{ll}\text { Methods } & 36\end{array}$

Design of study 36

$\begin{array}{ll}\text { Participants and setting } & 36\end{array}$

$\begin{array}{ll}\text { Procedure } & 37\end{array}$

$\begin{array}{ll}\text { Measures } & 37\end{array}$

$\begin{array}{ll}\text { Statistical analysis } & 37\end{array}$

$\begin{array}{ll}\text { Results } & 38\end{array}$

$\begin{array}{ll}\text { Discussion } & 40\end{array}$

$\begin{array}{ll}\text { HRQoL and coping } & 40\end{array}$

Coping modes after TBI $\quad 41$

Emotional adjustment and coping $\quad 41$

Clinical and socio-demographic aspects and coping 41

Predictors of coping $\quad 42$

Study limitations $\quad 42$

Conclusion $\quad 42$

References $\quad 42$

$\begin{array}{ll}\text { 6. DISKUSSION } & 44\end{array}$

6.1 Lebensqualität und Selbstwahrnehmung 45

6.2 Lebensqualität und Copingstrategien 46

6.3 Beurteilungsvalidität bei gestörter Selbstwahrnehmung 46

6.4 Kritische Anmerkungen und Limitationen 47

6.5 Fazit und Ausblick 49

7. ZUSAMMENFASSUNG 51

8. LITERATURVERZEICHNIS 53 


\section{EINLEITUNG}

Wie zufrieden bin ich mit meinen aktuellen Lebensumständen?

Wie beurteile ich meine Kompetenzen und Fähigkeiten?

Wie gehe ich mit Belastungen um?

Diese Fragen sind von zentraler Bedeutung für das menschliche Erleben und Verhalten. Antworten darauf geben die subjektive Perspektive des Betroffenen wider, d.h. seine individuelle Sichtweise. Zusammenhänge zwischen individuell-subjektiven Einschätzungen sind nicht nur von allgemeinpsychologischem Interesse, sondern auch in den anwendungsbezogenen Teildisziplinen der klinischen Psychologie und medizinischen Psychologie von besonderer praktischer Relevanz (wie z. B. zur Optimierung von Interventionen oder der Rehabilitation). In der vorliegenden Arbeit werden diese Zusammenhänge thematisiert und exemplarisch an einer Gruppe von Personen nach erlittenem Schädel-Hirn-Trauma (SHT) untersucht.

Das Erleiden eines Schädel-Hirn-Traumas (SHT) ist für jeden Betroffenen ein plötzliches Ereignis, das unvorhersehbar und unberechenbar aus dem alltäglichen Leben herausreißt und oftmals einschneidend gefasste Lebenspläne und -ziele durchkreuzt. Die SHT-Betroffenen werden mit ungeahnten Einschränkungen und Anforderungen konfrontiert und oft gezwungen, sich auf ein „neues“, stark verändertes Leben einzustellen. Diese neue Lebenssituation stellt meist eine kräftezehrende Herausforderung dar, die unmittelbar die Lebensqualität der Betroffenen tangiert.

Obgleich der Begriff „Lebensqualität“ erst kürzlich und spezifiziert als „gesundheitsbezogene Lebensqualität“ (gbzLQ) in die Medizin Eingang fand, stellt er heute ein etabliertes Zielkriterium für die Planung und Evaluation therapeutischer und rehabilitativer Maßnahmen dar, das neben der Funktionsfähigkeit auch das Wohlbefinden des Einzelnen aus seiner individuell subjektiven Sicht einbezieht (Steinbüchel et al., 2008). In einem übergreifenden Modell zur gbzLQ nach SHT (Steinbüchel, Petersen, Bullinger \& QOLIBRI Group, 2005a) werden Aspekte der Krankheitsverarbeitung und traumaspezifischen Selbstwahrnehmung der Betroffenen für eigene Kompetenzen und Fähigkeiten als 
relevante Faktoren identifiziert. Sie stellen im Gegensatz zu anderen spezifizierten Variablen, wie beispielsweise Persönlichkeits-, Lebenssituations- oder Unfallvariablen Einflussgrößen dar, die im Rahmen von rehabilitativen Maßnahmen modifiziert werden können. Damit eröffnet sich die Möglichkeit, die gbzLQ der Betroffenen gezielt zu verbessern.

In der klinischen Praxis wurde die Bedeutung der realitätsangemessenen Selbstwahrnehmung eigener Kompetenzen und einer adaptiven Krankheitsverarbeitung für den Rehabilitationserfolg und weiteren Krankheitsverlauf nach SHT bereits mehrfach beschrieben (Gauggel, Konrad \& Wietasch, 1998; Medley, Powell, Worthington, Chodan \& Jones, 2010; Prigatano, 1999/2004). Demgegenüber wurde die gbzLQ nach erlittenem SHT und deren Zusammenhänge mit der Selbstwahrnehmung und Krankheitsverarbeitung von SHT-Betroffenen bislang kaum oder nur unzureichend untersucht, da kein geeignetes Instrument zur krankheitsspezifischen Erfassung der gbzLQ nach SHT zur Verfügung stand. Darüber hinaus weist der gegenwärtige Forschungsstand zum Teil sehr unbefriedigende oder widersprüchliche Befunde in den Bereichen Selbstwahrnehmung und Krankheitsverarbeitung nach SHT auf.

Die vorliegende Arbeit möchte diese bislang nur lückenhaft erforschten Aspekte der gbzLQ nach traumatischer Hirnverletzung näher beleuchten und hier besonders die Zusammenhänge mit der Selbstwahrnehmung eigener Kompetenzen bzw. Fähigkeiten und der Krankheitsverarbeitung detaillierter untersuchen, um für die Planung und Durchführung von Rehabilitationsmaßnahmen weiterführende Erkenntnisse zur Verbesserung der Lebensqualität nach Schädel-Hirn-Trauma spezifizieren zu können. 


\section{FORSCHUNG ZU GESUNDHEITSBEZOGENER LEBENSQUALITÄT, SELBSTWAHRNEHMUNG UND KRANKHEITSVERARBEITUNG NACH SCHÄDEL-HIRN-TRAUMA}

\subsection{Schädel-Hirn-Trauma}

Die Deutsche Gesellschaft für Neurochirurgie definiert ein Schädel-Hirn-Trauma als „Folge einer Gewalteinwirkung, die zu einer Funktionsstörung und/oder Verletzung des Gehirns geführt hat und mit einer Prellung oder Verletzung der Kopfschwarte, des knöchernen Schädels, der Gefäße und/oder der Dura verbunden sein kann“ (AWMF online 2014, S2-Leitlinie Neurochirurgie: Schädel-HirnTrauma, S. 3). Diese eher weit gefasste Formulierung resultiert aus der gegenwärtig nicht bestehenden Übereinstimmung bezüglich einer international einheitlichen medizinischen Definition (Fearnside \& Simpson, 2005). In der klinischen Praxis erfolgt die Klassifikation eines SHT mittels des international anerkanntem ICD-10 Klassifikationssystems, das eine traumatische Hirnverletzung im Kapitel „Verletzungen des Kopfes“ unter S06. bis S06.9 codiert (AWMF online 2014; Fearnside \& Simpson, 2005). Tabelle 1 gibt einen Überblick über ICD-10-Kodierungen für SHT.

Tabelle 1: SHT-Klassifikation gemäß ICD-10

\begin{tabular}{ll} 
ICD-10-Kodierung & Diagnose \\
\hline S06.- & Intrakranielle Verletzung \\
S06.0 & Gehirnerschütterung \\
S06.1 & Traumatisches Hirnödem \\
S06.2 & Diffuse Hirnverletzung \\
S06.3 & Umschriebene Hirnverletzung \\
S06.4 & Epidurale Blutung \\
S06.5 & Traumatische subdurale Blutung \\
S06.6 & Traumatische subarachnoidale Blutung \\
S06.7 & Bewußtlosigkeit bei Schädelhirntrauma \\
S06.8 & Sonstige intrakranielle Verletzungen \\
S06.9 & Intrakranielle Verletzung, nicht näher bezeichnet
\end{tabular}

Zur Beurteilung des Schweregrades der traumatischen Hirnverletzung hat sich international die Glasgow Coma Scale (GCS; Teasdale \& Jennett, 1974) vergleichsweise gegenüber anderen Maßen 
wie der Dauer des Komas oder der posttraumatischen Amnesie etabliert (Lovasik, Kerr \& Alexander, 2001; Maas, Stocchetti \& Bullock, 2008). Bei einer innerhalb der ersten 24 Stunden nach erlittenem Trauma durchgeführten Vigilanz-Beurteilung der verunfallten Person (Öffnen der Augen, motorische und verbalen Reaktionen) stellt deren Gesamtpunktwert das zu klassifizierende Kriterium dar (3-8 Punkte: schweres SHT, 9-12 Punkte: mittelschweres SHT, 13-15: Punkte leichtes SHT). Kritisch wird das GCS-Kriterium allerdings für Fälle von Vigilanzverschlechterungen aufgrund neurologischer Komplikationen nach Ablauf des 24-Stunden-Intervalls gesehen. In der klinischen Forschung und Praxis werden derartige Fälle in einer zusätzlichen Kategorie als „leichtes SHT mit Komplikationen“ berücksichtigt, die einem mittelschweren oder schweren SHT-Verlauf entsprechen (Kashluba, Hanks, Casey \& Millis, 2008).

Die Ursachen mechanischer Krafteinwirkung auf das zentrale Nervensystem können unterschiedlicher Natur sein. Fearnside und Simpson (2005) unterscheiden hierzu eine verkehrsbedingte Verursachung durch Auto-, Motorrad-, Fahrrad- oder Fußgänger-Unfälle, eine sportbedingte Verursachung durch Reit-, Box-, Wintersport-, Inline- oder Skateboard-Unfälle sowie eine Verursachung durch Stürze, Alkohol- und Drogenkonsum oder gewalttätige Auseinandersetzungen mit oder ohne Waffeneinsatz. In Europa gelten Autounfälle zu den häufigsten Verursachungsgründen, Stürze zu den zweithäufigsten (Tagliaferri, Compagnone, Korsic, Servadei \& Kraus, 2006).

Aufgrund der sehr unterschiedlichen Ätiologie und untersuchten Populationen variieren die Inzidenzangaben für SHT. In einem Review europäischer Studien von Tagliaferri und Mitarbeitern (2006) wird eine jährliche Inzidenz von 235 SHT-Fällen und eine durchschnittliche Mortalitätsrate von ca. 15 Fällen auf jeweils 100000 Einwohner geschätzt. Die Schweregradverteilung (leichtes - mittelschweres - schweres SHT) wird hierbei mit $22: 1,5: 1$ angegeben. Aus einer prospektiven Studie werden für die Bundesrepublik Deutschland schätzungsweise 332 Fälle je 100000 Einwohner bei einer Sterblichkeit von 3,3 Fällen je 100000 Einwohner angegeben (Rickels, 2006b), wobei 90,9\% leichte, 3,9\% mittelschwere und 5,2\% schwere Schädel-Hirn-Verletzungen verzeichnet werden (Rickels, 2006a). Bedeutende Inzidenzfaktoren von Schädel-Hirn-Verletzungen sind Alter und 
Geschlecht. Junge Männer sind, vermutlich aufgrund erhöhter Risikobereitschaft, häufiger betroffen, wie auch ältere Personen aufgrund altersbedingt erhöhter Sturzgefahren (Kolb \& Whishaw, 1996; Maegele et al., 2007).

Je nach Art und Ausmaß der mechanischen Krafteinwirkung auf das Gehirn resultieren primäre und sekundäre Hirnverletzungen (Kiraly \& Kiraly, 2007; Maas et al., 2008). Primär entstehen Schädelfrakturen, makroskopische Läsionen und mikroskopische Läsionen durch Scherung und Zerrung von Nervenfasern und Zellmembranschäden. Sekundär resultieren daraus Hirnschwellung, eine Durchlässigkeitserhöhung der Blut-Hirn-Schranke, intrakraniale Druckerhöhung, intrakraniale, epidurale oder subarachnoidale Blutungen, Hirnödeme sowie Hirnverlagerung und Einklemmung. Trotz kompensatorischer Mechanismen des Gehirns und Spontanerholung (Kiraly \& Kiraly, 2007) führen diese Verletzungen oftmals zu irreversiblen Schädigungen, die mit funktionalen Beeinträchtigungen oder Ausfällen auf physischer, kognitiv-intellektueller und emotionaler Ebene (Jumisko, Lexell \& Söderberg, 2005; Lovasik et al., 2000) wie auch verhaltensbezogenen, sozialen und persönlichkeitsbezogenen Auffälligkeiten bzw. Schwierigkeiten verbunden sind. Diese können sich auf nahezu jeden Lebensbereich der Betroffenen, wie Arbeitsfähigkeit, Alltagsaktivität, Unabhängigkeit, Partnerschaft, soziale und gesellschaftliche Integration, Selbstbild und Selbstwertgefühl auswirken (Prigatano, 1999/2004; Truelle, Fayol, Montreuil \& Chevignard, 2010; Wood, Liossi \& Wood, 2005). Die durch das wechselseitige Zusammenspiel verschiedener hirnorganisch beeinträchtigter Funktionsbereiche bedingte Komplexität der SHT-Symptomatik wird als „neurobehavioural disability“ zu fassen versucht (Wood, 2013a,b).

Häufig berichtete Symptome von SHT-Betroffenen neben physischen Restriktionen und Hypopituitarismus (Behan \& Agha, 2007) betreffen kognitive Beeinträchtigungen in Aufmerksamkeit, Denkgeschwindigkeit, Gedächtnis, Konzentration, exekutiven Funktionen, Planungs- und Problemlösen sowie sprachlichen Funktionen (Kashluba et al., 2008; Krpen, Levine, Stuss \& Dawson, 2007; Navratil, Smrcka \& Hannak, 2006; Ponsford et al., 2014; Prigatano, 1999/2004; Skandsen et al., 2010), die Entwicklung psychiatrischer Erkrankungen mit vor allem Angststörungen und Depression, aber auch 
Posttraumatischer Belastungsstörung, Substanzmissbrauch und Persönlichkeitsstörungen (Corrigan \& Deutschle, 2008; Jorge, 2005; Schönberger, Ponsford, Gould \& Johnston, 2011; Warriner \& Velikonja, 2006), das Auftreten dysfunktionaler Verhaltensweisen wie Aggression bzw. Agitation, pathologisches Lachen und Weinen, Suizidalität (Baguley, Cooper \& Felmingham, 2006; Jorge, 2005; Lequerica et al., 2007; Lombard \& Zafonte, 2005; Tateno, Jorge \& Robinson, 2003) und weiterer Störungen wie rasche Erschöpfung (Cantor et al., 2008), Schwindel (Maskell, Chiarelli \& Isles, 2007) oder Schlafstörungen (Castriotta et al., 2007; Parcell, Ponsford, Redman \& Rajaratnam, 2008; Rao et al., 2008).

Rehabilitationsmaßnahmen nach SHT streben die möglichst vollständige Wiederherstellung der physischen und psychischen Funktionalität der SHT-Betroffenen an, dennoch können die Beschwerden über Jahre (Dikmen, Machamer, Powell \& Temkin, 2003; Whitnall, McMillan, Murray \& Teasdale, 2006) oder Jahrzehnte (Colantonio et al., 2004; Koponen et al., 2002) persistieren und zwingen oftmals die Betroffenen, ein „neues“ Leben mit Einschränkungen zu erlernen (Kasten, Gothe \& Müller, 2003).

Die Folgen einer erlittenen Hirnverletzung wurden vielfach anhand verschiedenster Kriterien untersucht (Jennett, 2005; Ponsford, 2013). Traditionell wurden hierbei Überlebensraten, physische Erholung, Invalidität, Unabhängigkeit bei Alltagsaktivitäten, Rückkehr zur Arbeit, gesellschaftliche Integration oder Teilhabe sowie klinische Störungen wie Angst oder Depression betrachtet. Aufgrund der eingeschränkten Anwendbarkeit einiger Kriteriumsmaße (wie z. B. Rückkehr zur Arbeit) wurden standardisierte Skalen zur Erfassung des funktionalen Outcome nach SHT, wie beispielsweise die Glasgow Outcome Scale (GOS; Jennett \& Bond, 1976) und ihre Erweiterung, die Glasgow Outcome Scale Extended (GOSE; Wilson, Pettigrew \& Teasdale, 1998) zur Einschätzung des körperlichen, sozialen und alltagsbezogenen Erholungsgrades nach SHT entwickelt (Jennett, 2005).

Bedingt durch den gewandelten Fokus auf patientennahe Zielkriterien in der Medizin (Bullinger, 2000) werden heute vermehrt „patient-reported outcomes measures“ (PROMs; Carlozzi, Tulsky \& 
Kisala, 2011; Herschbach, 2012; Meadows, 2011) als zusätzliche Maße für die Outcome-Beurteilung nach SHT verwendet, um die subjektive Perspektive der Betroffenen nach SHT einzuschließen. Hierzu zählen die subjektive Gesundheit oder die gesundheitsbezogene Lebensqualität (gbzLQ), die im folgenden Kapitel ausführlicher dargestellt wird.

\subsection{Gesundheitsbezogene Lebensqualität}

In die Medizin fand der Begriff Lebensqualität relativ spät, erst in den 70er Jahren Eingang. Ausschlaggebend hierfür war die WHO-Definition von Gesundheit (WHO, 1948), die nicht nur die Abwesenheit von Krankheit, sondern auch die psychischen und sozialen Aspekte von Wohlbefinden im Sinne eines biopsychosozialen Gesundheitsbegriffes berücksichtigte. Mit dieser Erweiterung des Gesundheitsbegriffes um psychosoziale Aspekte, dem gleichzeitig in dieser Zeit zu verzeichnenden Anstieg chronischer Erkrankungen und der damit verbundenen Notwendigkeit von neuen Zielkriterien sowie einem veränderten Gesundheitsbewusstsein mündig auftretender Patienten vollzog sich ein Paradigmenwechsel hinsichtlich relevanter Zielkriterien in der Medizin (Koch, 2000). Neben den bislang verwendeten klassischen biomedizinischen Indikatoren für den Gesundheitszustand, wie das Überleben oder die Reduktion von Symptomen, war seitdem auch ein patientennahes umfassenderes Kriterium von Interesse, das die individuell subjektive Gesundheitseinschätzung seitens des Betrof-fenen beinhaltete (Schumacher, Klaiberg \& Brähler, 2003). Das Bestreben, die subjektiv erlebte Gesundheit der Patienten nicht nur beiläufig im Arzt-Patienten-Gespräch zu tangieren, sondern sie konkret im wissenschaftlichen Sinne quantifizierbar zu machen, kennzeichnete den Eintritt der Erforschung von Lebensqualität (Bullinger, 2000, 2002) in einer zunehmend patientenorientiert ausgerichteten Medizin (Ravens-Sieberer \& Cieza, 2000).

Wissenschaftstheoretische Bemühungen, den Begriff Lebensqualität nominal zu definieren, führten bislang zu keinem einheitlichen Ergebnis (Casper, 1991; Herschbach, 2012). Demgegenüber wurde eine Vielzahl von operationalen Definitionen entwickelt (Kerekjarto, Schulz, Kramer, Fittschen \& Schug, 1989). Konsens besteht heute darin, dass das Konzept der Lebensqualität mindestens drei 
Bereiche (den physischen, psychischen und sozialen Lebensbereich) von Personen beinhaltet und als ein mehrdimensionales, relatives und vor allem subjektives Konstrukt mit einem dynamischen Charakter zu verstehen ist (Bullinger, 2000; Herschbach, 2012; Kerekjarto et al., 1989). In der internationalen Lebensqualitätsforschung hat sich die Definition gesundheitsbezogener Lebensqualität (gbzLQ) von Steinbüchel (1995; Steinbüchel et al., 2005a) etabliert. GbzLQ wird hierbei als ein multidimensionales Konstrukt verstanden, das körperliche, emotionale, mentale, soziale und alltagsbezogene Aspekte des Wohlbefindens sowie der Funktionsfähigkeit aus der subjektiven Perspektive des Betroffenen umfasst (Steinbüchel, 1995). Hierbei wird die betroffene Person als der beste Experte seiner selbst verstanden, dessen gbzLQ-Einschätzung im Falle schwerer erkrankungsbedingter Beeinträchtigung durch eine Fremdeinschätzung seitens eines beobachtenden Arztes oder Angehörigen ergänzt werden kann (Steinbüchel et al., 2010a).

Eine Abgrenzung des Lebensqualitätskonstrukts von anderen Konzepten wie dem subjektiven Gesundheitszustand, der Lebenszufriedenheit oder dem individuellen Selbstkonzept erscheint aufgrund der theoretisch mangelhaften Lebensqualitätsdefinition und seiner Dynamik erschwert und ist bis heute nicht eindeutig geklärt. Angenommen werden Überlappungen zwischen diesen Konzepten, wobei die gbzLQ als ein weiteres Konstrukt aufgefasst wird, dessen Eigenständigkeit als gesichert angenommen werden kann, da einzelne psychologische Prädiktoren kaum mehr als 50\% der Lebensqualitätsvarianz aufklären (Bullinger, 2000). Demgegenüber scheint das gbzLQ-Konzept interkulturell übertragbar und damit kulturübergreifend anwendbar zu sein.

Als Einflussfaktoren der Lebensqualität werden objektive Faktoren, wie die Erkrankungsschwere, Behandlungsintensität oder soziodemografische Variablen und subjektive Aspekte wie Persönlichkeitsmerkmale oder Anpassungskomponenten wie Krankheitsverarbeitung, Response Shift oder intraund interindividuelle Vergleichsprozesse gesehen, die die subjektiv-individuelle Beurteilung der gbzLQ beeinflussen (Herschbach, 2012). 
Zur Erfassung der gbzLQ wurden vielfältige Verfahren, wie offene Fragen, Interviews, psychometrische Fragebögen, individuelle Ansätze und gesundheitsökonomische Messinstrumente entwickelt. Im Gegensatz zu Persönlichkeitsinstrumenten sind Befragungsinstrumente zur gbzLQ für den Befragten transparent konstruiert, da die individuell subjektive Sicht des Betroffenen im Vordergrund steht. Im Bereich psychometrischer Fragebögen wurden sowohl krankheitsspezifische als auch generische oder krankheitsübergreifende Verfahren verwendet. Generische Verfahren wie z. B. der SF-36 (Ware \& Sherbourne, 1992) sind hierbei unabhängig von der untersuchten Erkrankung einsetzbar und ermöglichen Vergleiche zwischen Erkrankungspopulationen, besitzen jedoch nur Aussagekraft hinsichtlich allgemeiner Lebensqualitätsaspekte. Krankheitsspezifische Verfahren sind demgegenüber auf die Besonderheiten der Erkrankung zugeschnitten und ermöglichen eine differenzierte Aussage hinsichtlich der Lebensqualität innerhalb einer bestimmten Erkrankungspopulation. Weitgehend akzeptiert ist die Erfassung der gbzLQ im Selbstbeurteilungsverfahren, da die befragte Person als bester Experte der eigenen Befindlichkeit betrachtet wird (Steinbüchel et al., 2005a, 2008). Einschätzungen anderer Personen (Angehörige) werden nicht als Näherungswerte für die Lebensqualität des Befragten gesehen, sondern als ergänzende eigenständige Information. Für die Erfassung der gbzLQ spezifisch bei Personen nach erlittenem SHT konzipierten von Steinbüchel und Mitarbeiter (2005a) ein Messmodell, das die besonderen Aspekte der gbzLQ nach erlittener Hirnverletzung umfasst (s. Abb. 1). In dem spezifischen Messmodell von Steinbüchel und Mitarbeitern fließen neben den bereits von Herschbach (2012) genannten allgemeinen Einflussfaktoren, wie beispielsweise Copingstrategien, gleichfalls SHT-spezifische Faktoren, wie Beeinträchtigungen kognitiver und hier vor allem exekutiver Funktionen, Gedächtnisfunktionen sowie Störungen der Selbstwahrnehmung und des Selbstwertgefühls in die Beurteilung der gbzLQ nach SHT ein. 


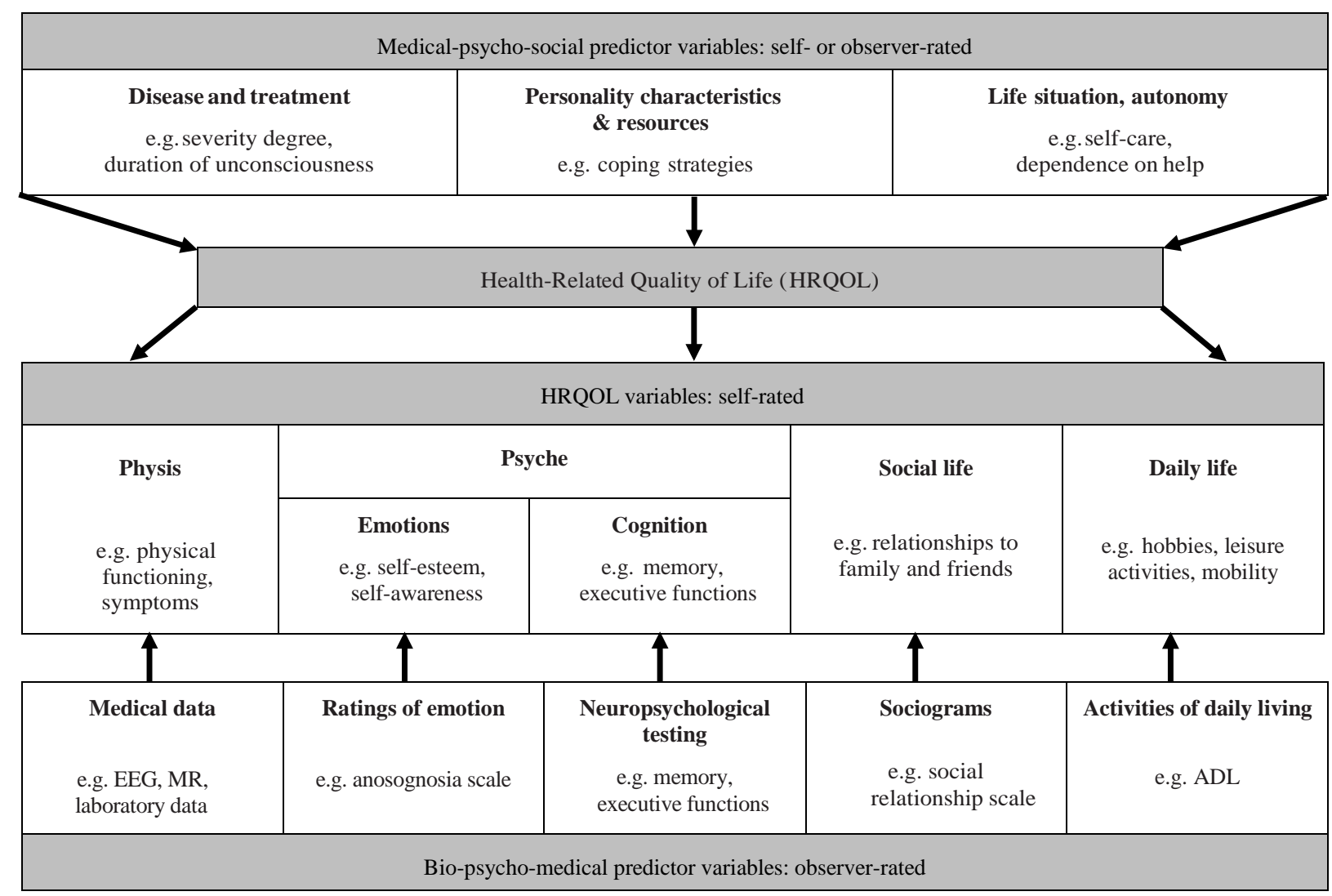

Abbildung 1: Messmodell gesundheitsbezogener Lebensqualität für Personen nach erlittenem Schädel-Hirn-Trauma (Original von Steinbüchel et al., 2005a)

Im Fall spezifischer Erkrankungspopulationen wie Betroffene nach erlittenem SHT wurde die Erfassung der gbzLQ im Selbstbericht lange Zeit stark problematisiert, da eine erkrankungsspezifische Selbstbeurteilung aufgrund eingeschränkter kognitiver Funktionalität angezweifelt wurde. Studienergebnisse mit SHT-Patienten widersprechen jedoch diesem Kritikpunkt und belegen in Subgruppenanalysen, dass eine reliable und valide Beurteilung der gbzLQ durch die Betroffenen auch bei kognitiver Beeinträchtigung durchaus möglich ist (Steinbüchel et al., 2008, 2010a,b).

Mit der Etablierung des neuen Zielkriteriums gbzLQ in der medizinischen Forschung und dessen Implementierung in die medizinische Praxis um die Jahrtausendwende hat das gbzLQ-Konzept zunehmend an Bedeutung gewonnen (Bullinger, 2002; Ravens-Sieberer \& Cieza, 2000; Steinbüchel et al., 2008). Es wird heute vielfach in Längs- oder Querschnittsstudien angewendet, u. a. beispielsweise in klinischen Studien zur Evaluation medizinischer Maßnahmen, in epidemiologischen Studien zur 
Bestimmung von gbzLQ-Verteilungen, in der Qualitätssicherung zur Beurteilung der Versorgungsqualität, in der Gesundheitsökonomie zur Bestimmung der adäquaten Mittelverteilung im Gesundheitswesen (Ravens-Sieberer \& Cieza, 2000) und auch in der medizinischen Routineversorgung zur Identifizierung subjektiver Erkrankungsanteile und Verbesserung der Kommunikation zwischen Arzt und Patient (Herschbach, 2012). Die erst vor wenigen Jahren erfolgte Entwicklung und Validierung des QOLIBRI-Fragebogens (Quality of Life after Brain Injury; Steinbüchel et al., 2010a,b, 2012) ermöglicht die detaillierte Erfassung und Untersuchung der SHT-spezifischen gbzLQ.

\subsection{Selbstwahrnehmung und gestörte Selbstwahrnehmung}

Die Auswirkungen eines Schädel-Hirn-Traumas betreffen in erster Linie das zentrale Nervensystem, das in seiner Funktion als Schalt- und Steuerungszentrale maßgeblich für die Funktionsfähigkeit des Individuums ist. Das Bewusstsein eines Menschen wird als die höchste integrative Hirnfunktion verstanden, die ihm eine objektive und realitätsgetreue Einschätzung seiner Selbst und seiner Umwelt ermöglicht (Prigatano, 1992, 1999/2004). Damit gilt das menschliche Bewusstsein als eine herausragende Hirnfunktion, deren Wirkungsweise aufgrund seiner Komplexität bis heute nicht eindeutig wissenschaftlich entschlüsselt wurde. Demgegenüber besteht nach Prigatano und Schacter (1991) Wissen darüber, dass das Bewusstsein eine objektive und eine subjektive Dimension beinhalten muss, die es dem Menschen ermöglicht, sowohl objektiv Kenntnis von einem Sachverhalt („knowledge of“) als auch Kenntnis über diesen Sachverhalt („knowledge with“) zu haben. Die Kenntnis über einen Sachverhalt gründet sich auf die im Laufe des Lebens gesammelten Erfahrungen, die jedem Individuum eine ihm innewohnende Theorie über seine eigene Funktionalität und daraus abgeleitete Erwartungen und Einschätzungen von Fähigkeiten für z. B. Handlungsabläufe ermöglicht (Goldenberg, 1997; Prigatano, 1992).

In diesem Zusammenhang schlagen Prigatano und Schacter (1991) folgende Definition von “consciousness or awareness“ vor: „Self-awareness is the capacity to perceive the „self“ in relatively „objective“ terms while maintaining a sense of subjectivity“ (S. 13) und betonen für dieses Konzept 
die Interaktion von Gedanken und Gefühlen. Hierbei kann beispielsweise eine Person mit eingeschränkter Gedächtnisfunktion über ihre Beeinträchtigung des Gedächtnisses durchaus Kenntnis besitzen, ohne sich jedoch deren funktionalen Auswirkungen im Alltag oder im Kontakt mit anderen Menschen bewusst zu sein.

Vor dem Hintergrund, dass das menschliche Bewusstsein individueller Kompetenzen und Fähigkeiten als eine der höchsten Hirnfunktionen verstanden wird, ist mit jeder Schädigung des zentralen Nervensystems, wie beispielsweise im Zuge eines SHT, eine Beeinträchtigung dieses Bewusstseins anzunehmen (Prigatano, 1999/2004). Die hieraus resultierende klinische Symptomatik von Betroffenen nach erlittener Hirnschädigung wurde bereits in der Antike beschrieben, ehe zu Beginn des 19. Jahrhunderts dieses Phänomen unter Verwendung verschiedener Bezeichnungen auch wissenschaftlich untersucht wurde. 1914 etablierte Babinski den Begriff „Anosognosie“ für das fehlende Bewusstsein einer Hemiplegie eines hirngeschädigten Patienten (Prigatano, 1999/2004; Prigatano \& Schacter, 1991). Der Begriff „Anosognosie“ entstammt hierbei dem Griechischen und umschreibt das Nichterkennen oder Nichtwahrnehmen einer Erkrankung, die durch Funktionsstörungen aufgrund der Hirnschädigungen bedingt sind (Goldenberg, 1997; Poeck, 1997).

In der wissenschaftlichen Erforschung dieses Phänomens werden bis heute vielfach verschiedene Termini synonym für die Umschreibung des Oberbegriffes „awareness“ oder „self-awareness“ (Krankheitseinsicht oder Störungsbewusstsein, aber auch Anosognosie, Krankheitsverleugnung, emotionale Indifferenz) genutzt (Gauggel et al., 1998), demgegenüber wird mit dem Begriff der „unawareness“ besonders das Fehlen der Bewusstseinsfähigkeit charakterisiert (Keller \& Grömminger, 1993). Beeinträchtigungen der „self-awareness“ werden in diesem Zusammenhang bedeutungsgemäß deutlich konsistenter benannt (Störung oder Einschränkung der Selbstwahrnehmung, Anosognosie (Keller \& Grömminger, 1993; Prigatano, 1999/2004), fehlendes bzw. vermindertes Störungsbewusstsein, fehlende bzw. verminderte Krankheitseinsicht oder Einsicht in eigene Defizite bzw. Leistungsfähigkeit (Gauggel et al., 1998; Keller \& Grömminger, 1993). 
Als klinischer Experte und maßgeblicher Forscher in diesem Bereich schlägt Prigatano folgende Definition eingeschränkter Selbstwahrnehmung für eigene Kompetenzen und Fähigkeiten nach erlittener Hirnschädigung im Sinne von Anosognosie vor: „...the clinical phenomena in which a brain dysfunctional patient does not appear to be aware of impaired neurological or neuropsychologial function, which is obvious to the clinician and other reasonably attentive individuals. The lack of awareness appears specific to individual deficits and cannot be accounted for by hyperarousal or widespread cognitive impairment.” (Prigatano, 1996, zitiert nach Prigatano, 2005, S. 20). Bezugnehmend auf diese Definition soll in der hier vorliegenden Arbeit für das subjektive Erkrankungsoder Störungsbewusstsein der Terminus „Selbstwahrnehmung“ verwendet werden, der die individuell eingeschätzten Kompetenzen und Fähigkeiten des SHT-Betroffenen in Relation zu einem Referenzkriterium, wie beispielsweise der Fähigkeitsbeurteilung durch nahe Angehörige umfasst. Zur Beschreibung von Einschränkungen des Bewusstseins für erkrankungsbedingte Beeinträchtigungen und Defizite soll weiterhin der Terminus „gestörte Selbstwahrnehmung“ im Sinne einer Anosognosie, d.h. der Überschätzung eigener Kompetenzen und Fähigkeiten seitens der SHT-Betroffenen verwendet werden.

In der zurückliegenden Forschung wurde die konzeptuelle Abgrenzung des Phänomens Anosognosie von psychologischer Krankheitsverleugnung („denial of illness“) vielfach diskutiert und kritisiert. Heute besteht Konsens darüber, dass im Erkrankungsverlauf nach einer Hirnschädigung beide Phänomene zum Tragen kommen können: sowohl eine Krankheitsverleugnung vor allem im frühen Stadium nach der erlittenen Hirnverletzung als sinnvoller psychologischer Abwehrmechanismus zur Stabilisierung des Selbst, als auch eine Störung der Selbstwahrnehmung des Betroffenen für eigene Kompetenzen und Fähigkeiten, die durch die hirnorganischen Schädigungen hervorgerufen wird (Gauggel et al., 1998).

Störungen der Selbstwahrnehmung treten sehr häufig nach traumatischen Hirnschädigungen auf (Bach \& David, 2006), verändern sich über die Zeit hinweg und können über Monate und Jahre persistieren bzw. chronifizieren (Prigatano, 1999/2004). In Abhängigkeit von der Lokalisation der Hirnläsionen 
kann sich das mangelnde Störungsbewusstsein auf eine Einzelfähigkeit oder auf einen gesamten Fähigkeitsbereich beziehen und tritt besonders häufig nach frontalen und rechtsseitigen Hirnschädigungen auf, was sehr eindrücklich die Beschreibungen über Anosognosie für Hemiplegie und linksseitige Hemiparese, Blindheit, Aphasie oder amnestische Syndrome verdeutlichen (Goldenberg, 1997).

Von Anosognosie betroffene Personen sind sich der aus dem erlittenen SHT resultierenden Beeinträchtigungen nicht bewusst und überschätzen dadurch ihre Kompetenzen und Fähigkeiten. Bei Befragung der Betroffenen werden Realitätsabweichungen mittels Verharmlosung, magischem Denken, bereits zuvor existierenden Defiziten oder ähnlichen Erklärungen begründet (Keller \& Grömminger, 1993) und überwiegend extern attribuiert oder bei teilweise intakter Selbstwahrnehmung mittels prämorbider Copingstrategien zu bewältigen versucht (Prigatano, 1999/2004).

Bislang existiert kein übergreifend gültiges wissenschaftliches Modell für diese Symptomatik. In einem Modell von Mesulam (1985, zitiert nach Prigatano, 1991) wird eine gestörte Selbstwahrnehmung als defizitäre Integration von primär afferenten Informationen und limbischen Informationen im heteromodalen Assoziationskortex beschrieben. Die kortikalen heteromodalen Assoziationsfelder werden hierbei als Basis der individuellen Selbstwahrnehmung angenommen, die die Zusammenführung von afferenten und affektiven Informationen ermöglichen. In diesem Modell wird die Selbstwahrnehmung nicht als eine rein kognitive Funktion aufgefasst, sondern vielmehr als höhere Hirnfunktion verstanden, die Denken und Fühlen sinnvoll vereint.

In der klinischen Praxis bedeutet dies für SHT-Betroffene, dass ihre bisherigen Theorien über ihre Kompetenzen und Fähigkeiten (basierend auf dem Erfahrungsschatz ihres Lebens vor der Hirnverletzung) nicht mehr zutreffen und diese korrigiert oder neu angelegt werden sollten (Goldenberg, 1997). Theorieabweichungen müssen hierzu zunächst von den Betroffenen erkannt werden, wobei diese im strukturellen Rahmen eines Krankenhauses aufgrund von Inaktivität im Krankenbett, fest organisierter Tagesabläufe, sozialer Strukturierungen etc. für den Betroffenen selbst kaum wahrnehmbar sind. Konfrontationen mit nunmehr beeinträchtigten Funktionsbereichen ergeben sich oftmals erst 
im alltäglichen Leben nach der Klinikentlassung, die dem Betroffenen die Erfahrung von funktionellen Veränderungen durch die Hirnverletzung ermöglichen und damit für ihn erschließbar werden.

In der Diagnostik von Störungen der Selbstwahrnehmung existieren mehrere Verfahrensweisen und unterschiedliche Instrumente für übergreifende Fähigkeitseinschätzungen oder unterschiedlich betroffene Funktionsbereiche. Gemeinsam ist den Verfahren, dass ein Referenzkriterium zur Fähigkeitsbeurteilung des SHT-Betroffenen benötigt wird. Hierzu werden Angehörigen-Beurteilungen, KlinikerBeurteilungen oder konkrete Testergebnisse verwendet (Keller \& Grömminger, 1993; Markova \& Berrios, 2006). Ein in der internationalen klinischen Praxis etabliertes Standardverfahren stellt die Patient Competency Rating Scale, PCRS (Prigatano et al., 1986) und deren Kurzform, die PCRS-NR (Borgaro \& Prigatano, 2003) dar, welche die Fähigkeitseinschätzungen allgemeiner Funktionsbereiche durch SHT-Betroffene im Vergleich zu der Fähigkeitsbeurteilung durch nahe stehende Angehörige erfassen. Als kritisch wird bei dieser Verfahrensweise ein möglicher Bias in der Funktionsbeurteilung durch die Angehörigen gesehen, etwa aufgrund von Verleugnung von vorhandenen Defiziten des zu betreuenden Verunfallten oder erhöhtem eigenem Distress durch die psychische Pflegebelastung (Fleming, Strong \& Ashton, 1996; Wise, Ownsworth \& Fleming, 2005). In ähnlicher Weise kann dies auch für Kliniker-Urteile zutreffen, wenn zu geringe Kenntnisse über die tatsächlichen Fähigkeiten und Defizite des Patienten vorhanden sind. Ein konkretes Testergebnis als Kriterium für die von dem Patienten zuvor eingeschätzten Fähigkeiten gilt als valideres Referenzmaß, allerdings ist dieses Messverfahren in klinischen Studien aus Kosten- und Zeitgründen selten realisierbar.

Störungen der Selbstwahrnehmung haben eine besondere Bedeutung für die Rehabilitation nach SHT. Das Erlernen eines „neuen“ Lebens mit den aus dem erlittenen Trauma resultierenden Einschränkungen ist nur möglich, wenn diese von dem Betroffenen bewusst erkannt, akzeptiert und in das zukünftige Leben integriert werden. Das Nichterkennen von funktionalen Beeinträchtigungen oder Behinderungen reduziert maßgeblich die Motivation eines SHT-Betroffenen, an einer Therapie teilzunehmen und sich darin zu engagieren, um an der Verminderung seiner Defizite zu arbeiten. Störungen der Selbstwahrnehmung sind aufgrund der mangelnden Compliance der SHT-Patienten als direktes 
Therapiehindernis einzuschätzen (Goldenberg, 1997; Schönberger, Humle, Zeeman \& Teasdale, 2006b). Für die Effektivität und das Outcome der Rehabilitation gelten sie damit als schlechtes prognostisches Zeichen, da sie dem Erlernen von kompensatorischen Strategien und dem Setzen realistischer Ziele entgegenwirken, so dass nach der Entlassung aus der Rehabilitation Überforderungen und Scheitern im Alltag zu erwarten sind (Goldenberg, 1997). In der Rehabilitation nach SHT ist es daher besonders wichtig, Störungen der Selbstwahrnehmung der Betroffenen so früh wie möglich zu identifizieren und zu behandeln. Es wird empfohlen, zeitgleich mit den rehabilitativen Interventionen ein Training zur Herstellung einer realistischen Selbstwahrnehmung durchzuführen, um den SHT-Betroffenen das Gewahrwerden ihrer Beeinträchtigungen und Defizite zu ermöglichen und damit die notwendige Motivation für die Mitarbeit bei therapeutischen Maßnahmen zu erlangen (Prigatano et al., 1986). Mit einer realitätsangemessenen Selbstwahrnehmung der SHT-Betroffenen für eigene Beeinträchtigungen und Defizite kann allerdings auch die emotionale Belastung zunehmen, die sich beispielsweise in erhöhten Angst- und Depressionswerten niederschlägt (Malec, Brown, Moessner, Stump \& Monahan, 2010; Malec, Testa, Rush, Brown, \& Moessner, 2007; Schönberger, Humle \& Teasdale, 2006a; Wallace \& Bogner, 2000). Eine Lösung für dieses Dilemma kann nicht darin bestehen, auf die Förderung des Gewahrwerdens von funktionalen Defiziten im Rahmen von rehabilitativen Maßnahmen zu verzichten, da sie die motivationale Voraussetzung für adaptive Lernprozesse und somit eine effektive Rehabilitation nach SHT darstellt (Prigatano et al., 1986, 1991). Vielmehr wird vorgeschlagen, ein vertrauensvoll lenkendes Arbeitsbündnis zwischen Therapeuten und Betroffenem zu etablieren (Schönberger et al., 2006a) und den SHT-Betroffenen mit wachsendem Gewahrwerden ihrer eigenen Defizite Strategien zu vermitteln, die ihnen eine positive Bewältigung der emotional belastenden Reaktionen ermöglichen (Prigatano et al., 1986).

Nur mit dem Erkennen und Akzeptieren der Defizite und Beeinträchtigungen durch die Hirnverletzung ist eine realistische Zielsetzung und Zukunftsplanung für ein verändertes und zufriedenstellendes Leben nach SHT möglich. Störungen der Selbstwahrnehmung stellen somit einen bedeutenden Einflussfaktor für die gbzLQ und damit für das Outcome nach erlittenem SHT dar (Dirette, 2002; Fischer, Gauggel \& Trexler, 2004; Halligan, 2006). 


\section{$2.4 \quad$ Coping und Krankheitsverarbeitung}

Die Forschung zu Coping bzw. Krankheitsverarbeitung basiert auf einer langen Tradition. Begründet durch den medizinischen Fortschritt wurden viele Erkrankungen besser behandelbar, was die Mortalitätsrate absinken ließ und zu einem drastischen Anstieg chronischer Krankheiten in der Medizin führte. Für die Betroffenen bedeutet dies zunehmend, mit den erkrankungsspezifischen Symptomen umgehen zu müssen oder gar ihr ganzes Leben darauf auszurichten. Die Klärung der therapeutischen Prozesse, in welcher Weise Menschen mit den Belastungen einer Erkrankung umgehen und welche Aspekte hierbei gesundheitsförderlich wirken, wurde zum Gegenstand der Coping-Forschung. Coping wird hierbei als Bewältigungsverhalten bezüglich einer akuten oder chronischen Erkrankung verstanden, wobei neben der erfolgreichen Bewältigung vielmehr die prozesshafte Verarbeitung der Symptomatik mit dem synonym genutzten Begriff „Krankheitsverarbeitung“ fokussiert wird (Kendel \& Sieverding, 2012).

Der Ursprung der Coping-Forschung liegt in der Stress-Forschung, wobei vor allem die Arbeiten von Selye (1976) zum allgemeinen Adaptationssyndrom (einer auf einen Auslöser folgenden reaktionsbezogenen 3-Phasen-Stressreaktion), die Life-Event-Forschung mit den Arbeiten von Holmes \& Rahe (1967) zur stimulusorientierten Stressreaktion in Abhängigkeit des Bedeutungsgehaltes des Stressauslösers und besonders der von Lazarus (1966) entwickelte Ansatz individueller innerpsychischer Bewertungsprozesse bei Stressreaktionen ausschlaggebend waren (Brüderl, Halsig \& Schröder, 1988; Kendel \& Sieverding, 2012). Das transaktionale Stressmodell von Lazarus und Folkman (1984) erlangte aufgrund seiner psychologischen Stresskonzeption und seiner ausführlichen theoretischen Ausarbeitung sowie empirischen Prüfung besondere Bedeutung im Kontext der Verarbeitung und Bewältigung verschiedenster Erkrankungen (Henze, 1994; Rüger, Blomert \& Förster, 1990).

In ihrer Konzeption gehen Lazarus und Folkman davon aus, dass die individuelle Wahrnehmung und subjektive Bewertung einer Situation durch eine Person unter Mitwirkung von persönlichen und situativen Faktoren zentral für die Auslösung einer Stressreaktion ist. Somit wird nicht mehr ein spezifi- 
scher Stimulus als stressauslösend auf einen Organismus angenommen, sondern vielmehr wird die Wechselwirkung zwischen Person und Umwelt als ausschlaggebend postuliert. Innerhalb des Wahrnehmungs- und Bewertungsprozesses einer Person wird eine „primäre Bewertung“ (Lazarus \& Folkman, 1984) vorgenommen, die als „irrelevant“, „angenehm-positiv“ oder „stressbezogen“ eingestuft werden kann. Bei Vorliegen einer stressbezogenen Bewertung der Situation werden in einer „sekundären Bewertung“ die zur Verfügung stehenden Handlungsmöglichkeiten und Ressourcen der Person zur Bewältigung der Situation evaluiert. Eine Situation wird schließlich als stressvoll erlebt, „...that is appraised by the person as taxing or exceeding his or her resources and endangering his or her well-being” (Lazarus \& Folkman, 1984, S. 19). Die Wahrnehmungs- und Bewertungsprozesse unterliegen nach Lazarus und Folkman einer Dynamik, was Neubewertungen von Situationen ermöglicht und besonders für den Verlauf chronischer Erkrankungen von Bedeutung sein kann. Zur Wiederherstellung des aus der Balance geratenen Gleichgewichts zwischen wahrgenommener Anforderungssituation und verfügbaren Ressourcen können von der Person verschiedene Bewältigungsstrategien mobilisiert werden, die entweder „problem-orientiert“ auf den Stressor direkt oder „emotionsorientiert“ auf die durch den Stressor ausgelöste emotionale Reaktion ausgerichtet sind (Lazarus \& Folkman, 1984). Problemorientierte Bewältigungsstrategien werden oft eingesetzt, wenn die Situation als veränderbar wahrgenommen bzw. bewertet wird, emotionsorientierte Strategien werden demgegenüber in Situationen eingesetzt, die als unveränderbar erlebt werden oder wenn die verfügbaren Ressourcen als nicht ausreichend beurteilt werden (Kendel \& Sieverding, 2012). In zahlreichen weiteren Arbeiten wurde diese international etablierte zweidimensionale Verarbeitungsstruktur durch differenziertere Dimensionierung oder Dimensionsumbenennung, wie z. B. aktive oder vermeidende Copingstrategien, verfeinert (Malia, Powell \& Torode, 1995).

Während Copingstrategien als überwiegend bewusst ablaufende Prozesse angesehen werden, sind die im Zusammenhang von Erkrankungen oftmals auftretenden psychoanalytischen Abwehrmechanismen wie Verleugnung oder Affekt-Isolierung als unbewusste Reaktionen zu differenzieren, die kurzfristig der Selbsterhaltung im Umgang mit massiven Ängsten und Bedrohungen in einer plötzlichen 
Krisensituation dienen können, eine bewusste Krankheitsverarbeitung langfristig jedoch erschweren (Henze, 1994).

Zur Erfassung von Coping entwickelten Folkman und Lazarus (1980) einen ihrer theoretischen StressKonzeption folgenden Fragebogen, die „Ways of Coping Checklist, WCCL“, die mit 68 Fragen Bewältigungsstrategien in einer als besonders stressreich wahrgenommenen allgemeinen Situation erfasst. Im Zuge der zunehmenden klinischen Relevanz von Coping-Prozessen im Kontext von Erkrankungen entstand ein vermehrter Bedarf an kontextspezifischen Erfassungsmethoden, da sich Instrumente der allgemeinen Coping-Forschung als wenig auf den klinischen Kontext übertragbar (nicht hinreichend dimensional oder sinnhaft) erwiesen (Muthny, 1989a). Mit der Entwicklung des Freiburger Fragebogens zur Krankheitsverarbeitung (FKV) übertrug Muthny (1989b) das transaktionale Stresskonzept von Lazarus und Folkman in den medizinischen Kontext klinischer Erkrankungen und ermöglichte dort erstmals dessen wissenschaftliche Erfassung. Muthny definierte Krankheitsverarbeitung als „...die Gesamtheit der Prozesse, um bestehende oder erwartete Belastungen im Zusammenhang mit Krankheit emotional, kognitiv oder aktional aufzufangen, auszugleichen oder zu meistern“ (Muthny, 1989b, S. 6). In seiner Konzeption betont Muthny neben dem gleichberechtigten Einfluss der kognitiven, emotionalen und sozialen Ebene auch den Prozesscharakter der Krankheitsverarbeitung, das Mitwirken von Persönlichkeits-, Situations- und Umweltfaktoren und die Einflussnahme des sozialen Umfeldes. Zusätzlich postulierte Muthny die verschiedenen Verarbeitungsmodi als unabhängig vom Verarbeitungserfolg, verbunden mit der Annahme, dass jede Person über ein gewisses Repertoire an verschiedenen Krankheitsverarbeitungsmodi verfügt, die in Abhängigkeit der individuell-subjektiven Situationswahrnehmung und -bewertung des Individuums von ihm eingesetzt werden. Im deutschsprachigen Raum stellt der FKV das am häufigsten angewendete Instrument zur Erfassung der Verarbeitung von Erkrankungen dar (Weis, 2008).

Zur Frage der Erkrankungsspezifität der Verarbeitung besteht bislang kein wissenschaftlicher Konsens. Muthny (1990) prüfte diese Fragestellung im Zuge der FKV-Entwicklung in Patientengruppen mit chronischer Niereninsuffizienz, koronarer Herzerkrankung und multipler Sklerose mit 
dem Ergebnis, dass die verwendeten Verarbeitungsstrategien sowohl durch erkrankungsübergreifende Gemeinsamkeiten als auch erkrankungsspezifische Unterschiede gekennzeichnet sind, und bestätigte damit frühere Befunde an repräsentativen Stichproben.

Im weiteren Forschungsverlauf wurden die Verarbeitungsprozesse bei einer Vielzahl von vor allem chronischen Erkrankungen, wie beispielsweise koronarer Herzerkrankung oder Krebs ausführlich untersucht (Kendel \& Sieverding, 2012). Bislang existieren jedoch nur wenige Studien zur Krankheitsverarbeitung nach erlittener Hirnverletzung, die die Art der verwendeten Verarbeitungsstrategien und deren Effektivität in Hinblick auf das Outcome (Gauggel et al., 1998) bzw. die Wirksamkeit rehabilitativer Interventionsstrategien zur Verbesserung von Verarbeitungsmodi nach SHT untersuchen (Krpan, Anderson \& Stuss, 2013).

Der stresstheoretische Ansatz von Lazarus und Folkman konnte bei SHT-Betroffenen zwar Eingang finden (Malia et al., 1995; Shotton, Simpson \& Smith, 2007) und in SHT-spezifischen CopingModellen, wie z. B. von Kendall und Terry (1996) oder Godfrey, Knight und Partridge (1996) weiterentwickelt werden, die Untersuchungsbefunde zu den von SHT-Betroffenen verwendeten Verarbeitungsstrategien erwiesen sich jedoch als sehr heterogen. Die heute existierende Vielfalt von krankheitsübergreifenden Coping-Instrumenten (und den damit erfassten unterschiedlichen Coping-Modi) erschweren deren Interpretation, da bislang kein SHT-spezifisches Instrument zur Erfassung der Krankheitsverarbeitung nach erlittener Hirnverletzung zur Verfügung steht. Nach Anson und Ponsford (2006) nutzen SHT-Betroffene aktive, interpersonale oder problem-orientierte Strategien, die sich positiv auf das Outcome nach SHT auswirkten und damit als adaptiv eingestuft wurden, während Flucht oder Vermeidung, passive oder emotionale Strategien einen ungünstigen Effekt in Bezug auf das psychosoziale Outcome sowie die emotionale Anpassung aufwiesen und damit als maladaptiv beurteilt wurden.

Es gilt als gesichert, dass die individuelle Art der Krankheitsverarbeitung als wichtige Einflusskomponente für das Outcome nach erlittener Hirnverletzung einzustufen ist (Anson \& Ponsford, 2006; Godfrey et al., 1996; Kendall \& Terry, 1996; Krpan et al., 2013; Malia et al., 1995). In dem bio- 
psycho-sozialen Modell der gesundheitsbezogenen Lebensqualität nach SHT von Steinbüchel und Mitarbeitern (2005a) werden Krankheitsverarbeitungsstrategien als wichtiger Parameter postuliert. Untersuchungen zum Zusammenhang der von SHT-Betroffenen eingesetzten Copingstrategien und deren Wirkung auf die Beurteilung der gbzLQ nach SHT sind allerdings kaum vorhanden, was auch hier durch den lange Zeit vorhandenen Mangel eines SHT-spezifischen Instrumentes zur Erfassung der gbzLQ mitbegründet sein kann. Die wenigen existenten Studien deuten auf einen Zusammenhang zwischen aktiv-problemorientiertem oder aufgabenorientiertem Coping und höheren gbzLQ-Beurteilungen (Tomberg, Toomela, Pulver \& Tikk, 2005; Wolters, Stapert, Brands \& Heugten, 2010) oder ungünstigen Änderungen der Strategieverwendung im SHT-Verlauf, die mit einer gbzLQ-Verschlechterung einhergehen (Tomberg, Toomela, Ennok \& Tikk, 2007), hin. 


\section{ZIELSETZUNG UND EIGENE FRAGESTELLUNGEN}

Im Rahmen der hier berichteten Arbeit sollen Lebensqualität, Einschätzungen der eigenen Kompe-tenzen und angewendete Copingstrategien als wichtige Aspekte des menschlichen Erlebens und Verhaltens aus der subjektiven Perspektive der Betroffenen beleuchtet werden. Hierzu wird die gesundheitsbezogene Lebensqualität (gbzLQ) exemplarisch bei Personen nach erlittenem Schädel-Hirn-Trauma (SHT) untersucht. Ziel ist es, mögliche Zusammenhänge zwischen der selbstbeurteilten gbzLQ des SHT-Betroffenen und den beiden, in der Rehabilitation gezielt modifizierbaren Faktoren Selbstwahrnehmung und Krankheitsverarbeitung $\mathrm{zu}$ überprüfen. In der SHT-Forschung gilt es als gesichert, dass das Outcome und auch die neueren „Patient Rated Outcome (PRO)“, wie beispielsweise die gbzLQ, als ein komplexes Gefüge verschiedener Einflussfaktoren zu verstehen sind. Es werden hierbei sowohl prämorbide, unfallspezifische, dem traumatischen Ereignis nachfolgende wie auch überdauernd einwirkende persönlichkeitsspezifische Faktoren angenommen, die in vermutlich unterschiedlichen Anteilen das Outcome nach erlittenem Trauma bedingen.

In einem mehrdimensionalen Messmodell der gbzLQ (Steinbüchel et al., 2005a) wurden diese Einflussfaktoren nach erlittenem SHT spezifiziert. Bereits vielfach untersucht und relativ gut belegt sind bislang Annahmen zum spezifischen Einfluss von traumabezogenen Merkmalen wie der Schwere des Traumas oder der Länge der posttraumatischen Amnesie, Aspekten der Lebenssituation wie z. B. die Unabhängigkeit von anderen Personen sowie der sozialen und gesellschaftlichen Einbindung. Demgegenüber ist die Bedeutung von personenspezifischen Ressourcen wie etwa Verarbeitungsstrategien beim Umgang mit den Traumafolgen in Hinblick auf die gbzLQ nach SHT unzureichend wissenschaftlich evaluiert. Auch zum Einfluss von Selbstwahrnehmung und Störungen der Selbstwahrnehmung auf die gbzLQ nach SHT existieren widersprüchliche Befunde.

Obgleich die Bedeutung einer vorliegenden Störung der Selbstwahrnehmung bzw. Anosognosie wie auch die Art der Krankheitsverarbeitung für die Rehabilitation in klinischen Studien herausgestellt wurde, konnte bislang ein möglicher Zusammenhang mit der SHT-spezifischen gbzLQ kaum geklärt 
werden. Erst seit kurzem steht mit dem QOLIBRI-Fragebogen (Steinbüchel et al., 2010a,b, 2012) für diese Betroffenengruppe ein Instrument zur Erfassung der spezifischen gbzLQ zur Verfügung und ermöglicht erstmals die detaillierte Untersuchung dieses komplexen krankheitsspezifischen Konstruktes. Richtung und Ausprägung möglicher Zusammenhänge zur SHT-spezifischen gbzLQ können nun nicht nur global, sondern auch differenziert nach unterschiedlichen Inhaltsbereichen geprüft werden.

In den folgenden Kapiteln wird die SHT-spezifische gbzLQ fokussiert, vergleichsweise der allgemeinen gbzLQ gegenübergestellt und der Bedeutungsgehalt der beiden, auch klinisch relevanten Einflussfaktoren Selbstwahrnehmung und Krankheitsverarbeitung detailliert untersucht.

In der ersten Studie (Sasse et al., 2013; siehe Kapitel 4) wird die gbzLQ nach SHT in Verbindung mit der relativen Selbstwahrnehmung der eigenen Kompetenzen nach dem erlittenen Trauma betrachtet. Die Fragestellung ist hier, welcher Zusammenhang zwischen der Selbstwahrnehmung und der globalen gbzLQ sowie deren bereichsspezifischen Ausprägungen besteht. Eine weitere Frage ist, ob sich SHT-Betroffene mit intakter vs. gestörter relativer Selbstwahrnehmung hinsichtlich ihrer angegebenen gbzLQ und in weiteren relevanten Merkmalen unterscheiden. Darüber hinaus wird den Fragen nachgegangen, welche Zusammenhänge zwischen der relativen Selbstwahrnehmung der Betroffenen mit wieteren klinischen und soziodemografischen Aspekten bestehen, und ob sich Prädiktoren der Selbstwahrnehmung nach SHT identifizieren lassen.

In der zweiten Studie (Sasse et al., 2014; siehe Kapitel 5) wird die gbzLQ nach SHT im Zusammenhang mit der Art der Krankheitsverarbeitung aufgegriffen. Im Vordergrund steht dabei die Frage, welche Krankheitsverarbeitungsmodi nach erlittenem SHT von den Betroffenen genutzt werden, um die Folgen der Hirnverletzung zu bewältigen. Auch soll die Effektivität der von den Betroffenen eingesetzten Verarbeitungsstrategien in Hinblick auf das Outcome nach dem Trauma geprüft werden. Abschließend wird auch hier der Frage nachgegangen, inwiefern Prädiktoren der Verarbeitungsstrategien nach SHT zu identifizieren sind. 


\section{SELBSTWAHRNEHMUNG UND GESUNDHEITSBEZOGENE LEBENSQUALITÄT NACH SCHÄDEL-HIRN-TRAUMA}

Dieses Kapitel (S. 25-33) beinhaltet das beim amerikanischen „Journal of Head Trauma and Rehabilitation“ publizierte Manuskript: Sasse, N., Gibbons, H., Wilson, L., Martinez-Olivera, R., Schmidt, H., Hasselhorn, M., Wild, K. von \& Steinbüchel, N. von (2013). Self-awareness and health-related quality of life after traumatic brain injury. Journal of Head Trauma and Rehabilitation, 28, 464-472 (published ahead-of-print August 29, 2012). 
http://www.ncbi.nlm.nih.gov/pubmed/22935572 
http://www.ncbi.nlm.nih.gov/pubmed/22935572 
http://www.ncbi.nlm.nih.gov/pubmed/22935572 
http://www.ncbi.nlm.nih.gov/pubmed/22935572 
http://www.ncbi.nlm.nih.gov/pubmed/22935572 
http://www.ncbi.nlm.nih.gov/pubmed/22935572 
http://www.ncbi.nlm.nih.gov/pubmed/22935572 
http://www.ncbi.nlm.nih.gov/pubmed/22935572 
http://www.ncbi.nlm.nih.gov/pubmed/22935572 


\section{KRANKHEITSVERARBEITUNGSSTRATEGIEN VON PERSONEN NACH SCHÄDEL-HIRN-TRAUMA: ZUSAMMENHÄNGE MIT GESUNDHEITSBEZOGENER LEBENSQUALITÄT}

Dieses Kapitel (S. 35-43) beinhaltet das beim britischen Journal „Disability and Rehabilitation“ eingereichte und publizierte Manuskript: Sasse, N., Gibbons, H., Wilson, L., Martinez, R., Sehmish, S., Wild, K. von \& Steinbüchel, N. von (2014). Coping strategies in individuals after traumatic brain injury: associations with health-related quality of life. Disability and Rehabilitation, March 3 early online: 1-9, DOI: 10.3109/09638288.2014.893029. 
http://www.ncbi.nlm.nih.gov/pubmed/24579650 
http://www.ncbi.nlm.nih.gov/pubmed/24579650 
http://www.ncbi.nlm.nih.gov/pubmed/24579650 
http://www.ncbi.nlm.nih.gov/pubmed/24579650 
http://www.ncbi.nlm.nih.gov/pubmed/24579650 
http://www.ncbi.nlm.nih.gov/pubmed/24579650 
http://www.ncbi.nlm.nih.gov/pubmed/24579650 
http://www.ncbi.nlm.nih.gov/pubmed/24579650 
http://www.ncbi.nlm.nih.gov/pubmed/24579650 


\section{DISKUSSION}

In der hier vorliegenden Arbeit wurden Zusammenhänge zwischen verschiedenen Aspekten der subjektiv erlebten Lebenssituation untersucht, um daraus anwendungsrelevante, aber auch allgemeinpsychologisch interessierende Aussagen abzuleiten. Hierzu wurde eine Gruppe von Personen nach erlittenem Schädel-Hirn-Trauma (SHT) gebeten, ihre individuelle Lebensqualität und ihre eigenen Fähigkeiten bzw. Kompetenzen zu beurteilen sowie Fragen zu den von ihnen angewendeten Verarbeitungsstrategien zu beantworten.

Durch den Umstand, dass lange Zeit kein Instrument zur Erfassung der SHT-spezifischen gesundheitsbezogenen Lebensqualität (gbzLQ) nach dem erlittenem Trauma zur Verfügung stand, konnten bislang nur globale und unspezifische Angaben zur subjektiven Zufriedenheit und Befindlichkeit dieser Betroffenengruppe ermittelt werden. Erst mit der Entwicklung und Validierung des QOLIBRI-Fragebogens (Steinbüchel et al., 2010a,b, 2012) wurde die SHT-spezifische Messung der spezifischen Lebensqualität ermöglicht. Diese Innovation wurde in der vorliegenden Forschungsarbeit aufgegriffen und eingebunden, so dass erstmals speziell auf diese Betroffenengruppe bezogene und für unterschiedliche Inhaltsbereiche differenzierte Forschungsergebnisse zur Lebensqualität erbracht werden konnten. Darüberhinaus war es nun auch möglich, die subjektiv erlebte Lebensqualität der TraumaBetroffenen in Hinblick auf ihre Beziehungen zu den zwei klinisch wie auch allgemeinpsychologisch relevanten Merkmalen, Selbstwahrnehmung bzw. gestörte Selbstwahrnehmung für eigene Kompetenzen und Fähigkeiten und den individuell eingesetzten Verarbeitungsstrategien gemäß dem SHTspezifischen gbzLQ-Modell von Steinbüchel und Mitarbeitern (2005a) zu untersuchen.

Die Ergebnisse der dieser Arbeit zugrunde liegenden zwei Publikationen zeigen, dass die relative Selbstwahrnehmung von Betroffenen nach einem erlittenen Trauma in Beziehung zu der subjektiven Beurteilung ihrer gbzLQ steht. Auch bestätigen die Befunde dieser Arbeit, dass ein Zusammenhang zwischen der Art der Verarbeitung des erlebten Traumas und der subjektiv empfundenen Lebensqualität nach dem Trauma besteht. 


\subsection{Lebensqualität und Selbstwahrnehmung}

In der ersten Studie wird gezeigt, dass eine (im Vergleich zu den Angaben ihrer Angehörigen) relativ positivere Selbstwahrnehmung von Betroffenen mit der Einschätzung ihrer gbzLQ positiv assoziiert ist. Dies bedeutet, dass SHT-Betroffene mit einer beeinträchtigten Selbstwahrnehmung, d. h. einer überhöhten Einschätzung eigener Kompetenzen und Fähigkeiten auch ihre gbzLQ positiver beurteilen. Diese positivere Einschätzung der gbzLQ ist besonders für den Bereich der geistigen Fähigkeiten und dem Selbstbild deutlich. Der soziale, emotionale und physische gbzLQ-Bereich wird tendenziell ebenfalls als zufriedenstellender beurteilt, während die alltagsbezogene gbzLQ eher weniger positiv eingeschätzt wird. Der direkte Vergleich von Untergruppen von SHT-Betroffenen mit und ohne beeinträchtigter Selbstwahrnehmung konnte diesen Effekt nur tendenziell untermauern, dennoch unterstreicht die Gruppenanalyse ebenfalls die vorrangige Bedeutung des kognitiven Bereichs der Lebensqualität für den Personen mit einer gestörten, überhöht positiven Selbstwahrnehmung eine stärkere Zufriedenheit berichteten.

Darüber hinaus wurden zwischen der relativen Selbstwahrnehmung der Betroffenen und ihren Angstund Depressionswerten negative Assoziationen ermittelt, welche das bereits in früheren Studien berichtete Dilemma veranschaulicht: eine realistischere Selbstwahrnehmung SHT-bedingter Defizite geht einher mit einer gleichfalls steigenden emotionalen Belastung. Die gefundene positive Assoziation mit der Schwere des erlittenen Traumas belegt ein vermehrtes Auftreten von Störungen der Selbstwahrnehmung nach mittelschweren bis schweren Hirnverletzungen. Ein vorhersagekräftiger einzelner Prädiktor der relativen Selbstwahrnehmung wurde nicht identifiziert, varianzanalytisch zeigten sich allerdings Interaktionseffekte zwischen Geschlecht und Alter sowie Geschlecht und Unabhängigkeit von anderen Personen, die als Hinweise für in weiterführenden Studien möglicherweise zu prüfende Prädiktoren eingestuft werden können. 


\subsection{Lebensqualität und Copingstrategien}

In der zweiten Studie zum Einfluss der Krankheitsverarbeitung auf die Beurteilung der SHT-spezifischen gbzLQ konnte gezeigt werden, dass von den Trauma-Betroffenen vorrangig zwei Verarbeitungsstrategien genutzt werden, um mit den Folgen eines erlittenen SHTs umzugehen: Trivialisierung/Resignation und Aktivität/Ablenkung. Diese Strategien stehen in jeweils unterschiedlichem Zusammenhang mit der gbzLQ-Beurteilung und besitzen damit auch eine klinische und alltagsbezogene Bedeutung. Hierbei ist die erstgenannte Strategie der Trivialisierung/Resignation als eine maladaptive Strategie einzuschätzen, da sie in deutlich negativem Zusammenhang mit allen gbzLQBereichen und in positivem Zusammenhang mit ungünstigen klinischen Variablen wie Angst, Depression, Müdigkeit und Ärger steht. Demgegenüber scheint die Strategie der Aktivität/Ablenkung eine sehr spezifische und adaptive Strategie zu sein, da sie in positivem Zusammenhang mit umschriebenen Bereichen der gbzLQ (Selbstbild und soziale Beziehungen) steht und für diese Strategie keine Assoziationen mit den genannten ungünstigen klinischen Variablen gefunden wurden. Für die Verwendung der Strategie Trivialisierung/Resignation seitens der SHT-Betroffenen scheint der funktionale Status bzw. der Behinderungsgrad ausschlaggebend zu sein, da dieser als relevanter Prädiktor für diese maladaptive Verarbeitungsstrategie identifiziert wurde.

\subsection{Beurteilungsvalidität bei gestörter Selbstwahrnehmung}

Der in der ersten Studie ermittelte positive Zusammenhang zwischen der relativen Selbstwahrnehmung der Betroffenen und ihrer gbzLQ-Beurteilung stellt ein erwartungskonformes Ergebnis dar: Im Falle einer gestörten Selbstwahrnehmung (d. h. bei überhöht positiver Selbsteinschätzung eigener Kompetenzen und Fähigkeiten) ist bei zunehmender Fähigkeitsüberschätzung seitens des Betroffenen gleichfalls auch eine Erhöhung seiner Lebensqualitätsbeurteilung zu erwarten. Speziell in derartigen Fällen mit überhöhter Selbsteinschätzung der eigenen Kompetenzen ist eine eingeschränkte Validität von gbzLQ-Beurteilungen zu befürchten.

Dies bedeutet nicht etwa, dass die gbzLQ-Messung generell als unzureichend valide zu kritisieren wäre: Überprüfungen des QOLIBRI-Fragebogens bestätigen dessen Validität als Instrument zur 
Erfassung der gbzLQ nach SHT und konnten in Subgruppenanalysen auch die Möglichkeit mangelnder Validität bei hirnverletzungsbedingten kognitiven Beeinträchtigungen von SHT-Betroffenen ausschließen (Steinbüchel et al., 2010a,b; 2014). Aus dem Blickwinkel der gbzLQ als Schätzwert für das Outcome nach SHT wird die Beantwortung der aufgeworfenen Validitätsfrage in Fällen gestörter Selbstwahrnehmung (Anosognosie) jedoch schwieriger, da mit einer Erhöhung der betrachteten Zufriedenheit auch eine Verzerrung des Informationsgehaltes der gbzLQ-Werte als subjektive Ergänzung objektiver Outcome-Werte anzunehmen ist.

Das Konzept der gbzLQ basiert auf der subjektiven Betroffenenperspektive (Steinbüchel, Richter, Morawetz \& Riemsma, 2005b), das den SHT-Betroffenen als besten Experten seiner selbst versteht und somit die Angaben des Befragten per definitionem als gültige gbzLQ-Werte zu verstehen sind. GbzLQ stellt dadurch ein subjektives Outcome-Kriterium dar, das keinen Anspruch auf objektive Gültigkeit anstrebt. Wenn in diesem Zusammenhang ein Vergleich mit objektiven Outcome-Kriterien nach SHT angestrebt sein sollte, ist bei SHT-Betroffenen mit einer Störung der Selbstwahrnehmung bzw. Anosognosie der zusätzliche diagnostische Wert der selbsteingeschätzten gbzLQ mittels des QOLIBRI-Fragebogens nur mit Vorbehalt gegeben. Die generelle Validität des QOLIBRI-Fragebogens könnte durch die ergänzende zusätzliche Erfassung und Berücksichtigung einer möglicherweise vorliegenden Störung der Selbstwahrnehmung auch bei diesen Patienten erhöht werden.

\subsection{Kritische Anmerkungen und Limitationen}

Für die Messung von Selbstwahrnehmung bzw. Störungen der Selbstwahrnehmung existieren mehrere diagnostische Verfahrensweisen, die sich vorwiegend in ihren verwendeten Referenzkriterien für die Betroffenen-Einschätzung unterscheiden. Hierbei wäre (wie in Abschnitt 2.3 dargestellt) abzuwägen, inwiefern die Beurteilung der eigenen Kompetenzen durch den SHT-Betroffenen mit den Einschätzungen seiner Angehörigen (wie in der Patient Competency Rating Scale in der vorliegenden Arbeit geschehen) verglichen wird, oder ob nicht eher der Vergleich der Selbsturteile der Betroffenen mit objektiven Leistungsdaten vorteilhafter und valider wäre. 
Das Erleiden eines SHT ist nicht notwendigerweise mit einer einhergehenden Störung der Selbstwahrnehmung verbunden. Diese Symptomatik der Selbstüberschätzung tritt in Abhängigkeit spezifisch betroffener Hirnareale oder diffuser axonaler Schädigungen auf, was sich vermutlich in der geringen Höhe der gefundenen positiven Assoziationen zwischen der relativen Selbstwahrnehmung und der gbzLQ-Beurteilung der Betroffenen niederschlägt.

Insgesamt sind die in beiden Publikationen betrachteten Beziehungen der untersuchten Einflussgrößen und der SHT-spezifischen gbzLQ durch überwiegend schwache Zusammenhänge gekennzeichnet. Dies lässt auf eine nur begrenzte Varianzaufklärung der untersuchten Variablen in dem komplexen Messmodell der SHT-spezifischen gbzLQ schließen.

In beiden hier vorgelegten Veröffentlichungen wurde methodisch sowohl ein kategorialer Ansatz (mittels einer Gruppenanalyse zur Differenzierung zwischen SHT-Betroffenen mit und ohne Störung der Selbstwahrnehmung sowie einer Gruppierung von Verarbeitungsstrategien nach SHT) als auch ein korrelativer Ansatz gewählt, um die Beziehungen zwischen den betrachteten Einflussvariablen und der berichteten gbzLQ nach SHT zu untersuchen. Zur Untersuchung der zentralen Fragestellung dieser Arbeit wurden somit vornehmlich korrelative Zusammenhänge zwischen der relativen Selbstwahrnehmung, den Krankheitsverarbeitungsstrategien und der globalen gbzLQ sowie auf den verschiedenen gbzLQ-Bereichen betrachtet, die keine kausalen Aussagen über die identifizierten Assoziationen zulassen. Diesem interpretativen Mangel könnte in weiterführenden Studien beispielsweise mit pfadanalytischen Auswertungsmethoden begegnet werden.

Eine Einschränkung der hier berichteten eigenen Forschungsarbeiten stellt die Stichprobenzusammensetzung dar. Beide Untersuchungen erfolgten im Rahmen eines internationalen Projekts zur Entwicklung und Validierung des QOLIBRI-Fragebogens (Steinbüchel et al., 2010a,b). Die Studienteilnehmer wurden konsekutiv angeschrieben, konnten aber keine finanzielle Aufwandsentschädigung für ihre Teilnahme erwarten, so dass ein Selektionsbias in Bezug auf besonders motivierte Studienteilnehmer mit gutem Erholungsstatus zu vermuten ist. In diesem Zusammenhang ist gleichfalls die 
Stichprobengröße kritisch zu erwähnen, die sich maßgeblich durch den für die internationale Studie erforderlichen Umfang bedingte und durch die gesetzten Einschlusskriterien (z. B. die Notwendigkeit eines vorliegenden GCS und GOSE-Wertes, vgl. Kapitel 4 und 5) weiter reduzierte. Die resultierende Analysestichprobe ist möglicherweise insgesamt nur als bedingt repräsentativ einzuschätzen.

Auch ist das innerhalb der internationalen Validierungsstudie vorgegebene, sehr weit gefasste Zeitintervall zwischen dem erlittenen Trauma und dem Befragungszeitpunkt der SHT-Betroffenen zu nennen, das sich für die hier untersuchten Fragestellungen als weniger günstig erweisen könnte, da sowohl Einschränkungen der Selbstwahrnehmung als auch Krankheitsverarbeitungsstrategien einem Wandel über die Zeit unterliegen und dies möglicherweise in der Gesamtbetrachtung zur Reduzierung oder Nivellierung der hier geprüften Effekte geführt haben könnte.

\section{5 $\quad$ Fazit und Ausblick}

In der vorliegenden Arbeit wurden zwei im SHT-spezifischen gbzLQ-Modell (von Steinbüchel et al., 2005a) enthaltene Determinanten der gbzLQ herausgegriffen und gesondert betrachtet, um deren jeweiligen Einfluss detailliert zu überprüfen. Auf die hier gewonnenen Erkenntnisse zum bedeutsamen Einfluss von Selbstwahrnehmung bzw. gestörter Selbstwahrnehmung und der Art der Krankheitsverarbeitung auf die gbzLQ aufbauend, könnte eine Zielsetzung zukünftiger Forschung darin bestehen, diese nicht als unabhängige Einzelparameter im Modell der spezifischen gbzLQ nach erlittenem SHT zu betrachten, sondern auch deren wechselseitigen Zusammenhang zu prüfen. In einer Studie von Medley und Mitarbeitern (2010) konnte im Rahmen eines Selbstregulationsmodells bereits das Zusammenspiel dieser beiden Merkmale mittels eines clusteranalytischen Ansatzes erfolgreich gezeigt werden, indem drei verschiedene SHT-Betroffenengruppen mit unterschiedlichen Ausprägungen relativer Selbstwahrnehmung und verschiedenen präferierten Krankheitsverarbeitungsstrategien identifiziert wurden. Auch weist eine Studie von Lundqvist und Alinder (2007) auf einen möglichen komplexen Zusammenhang zwischen der relativen Selbstwahrnehmung und Coping-Verhalten am Beispiel des Autofahrens hin, wobei die Autoren die Rolle von Metakognitionen und exekutiven 
Funktionen betonen. Darüber hinaus könnte in zukünftiger Forschung der Fragestellung nachgegangen werden, welchen individuellen Beitrag die in dieser Arbeit herausgegriffenen und jeweils einzeln betrachteten Einflussfaktoren im umfassenderen Kontext aller im Modell enthaltenen Merkmale leisten.

Die hier vorgelegte Forschungsarbeit möchte mit der Untersuchung der zwei Einflussgrößen Selbstwahrnehmung bzw. gestörte Selbstwahrnehmung und Krankheitsverarbeitung im Rahmen des SHTspezifischen Modells der gbzLQ erste Ergebnisse zur Verfügung stellen. Eine kritisch-systematische Evaluation der erbrachten Befunde ist aufgrund der gegenwärtig kaum vorhandenen vergleichbaren Studienergebnisse zum Einfluss dieser Variablen auf die spezifische gbzLQ nach erlittenem SHT erschwert. Hierin zeigt sich ein zukünftiger Forschungsbedarf, deren Beginn mit diesen Arbeiten angestoßen werden sollte. Sie können als erste Hinweise für die Prüfung weiterführender spezifischer Fragestellungen innerhalb des theoretisch formulierten Modells der gbzLQ dienen und dadurch zur Optimierung der Diagnostik und Rehabilitation der betroffenen SHT-Patienten beitragen.

Die in der vorliegenden Forschungsarbeit untersuchten Merkmale und Zusammenhänge sind über die hier im Vordergrund thematisierte klinisch-praktische Anwendung hinaus auch von allgemeinpsychologischem Interesse, da sie im individuellen Alltagsleben wiederkehrende elementare Aspekte des menschlichen Erlebens und Verhaltens betreffen: die Zufriedenheit mit den eigenen Lebensumständen, die Einschätzung der eigenen Fähigkeiten und des aktuellen Leistungsvermögens und die präferierten Strategien im Umgang mit alltäglichen Herausforderungen, Einschränkungen und Belastungen. 


\section{ZUSAMMENFASSUNG}

Die individuell-subjektive Perspektive bei Beurteilungen der eigenen Lebenssituation ist nicht nur von allgemeinpsychologischem Interesse, sondern besitzt auch eine besondere anwendungsorientierte Relevanz in klinisch-psychologischen und medizinischen Bereichen. So ist die gesundheitsbezogene Lebensqualität (gbzLQ) ein etabliertes Outcome-Kriterium in der medizinischen Versorgung, das objektive diagnostische Indikatoren um die von Patienten subjektiv erlebte und beurteilte Gesundheit und Zufriedenheit ergänzt. Erst seit kurzem kann dieses Kriterium auch bei Personen nach erlittenem Schädel-Hirn-Trauma (SHT) Anwendung finden, da lange Zeit für diese Betroffenengruppe kein Instrument zur Erfassung der spezifischen gbzLQ zur Verfügung stand. Dieser Mangel wurde mit der Entwicklung und Validierung des QOLIBRI-Fragebogens behoben, der in dieser Forschungsarbeit innovativ Anwendung findet. Damit eröffnet sich auch die Möglichkeit, gemäß dem gbzLQ-Messmodell von Steinbüchel und Mitarbeitern (2005a) weitere mit der spezifischen gbzLQ zusammenhängende anwendungsrelevante Aspekte wie die Selbstwahrnehmung von Betroffenen nach erlittenem SHT und deren individuellen Verarbeitungsstrategien des Traumas zu beleuchten.

Ziel dieser Forschungsarbeit ist daher die Untersuchung der erkrankungsspezifischen gbzLQ von SHT-Betroffenen im Kontext der Einflussgrößen relative bzw. gestörte Selbstwahrnehmung und zur Krankheitsverarbeitung verwendeter Coping-Strategien.

Die Ergebnisse der hier berichteten eigenen Untersuchungen belegen deutliche Zusammenhänge zwischen der relativen Selbstwahrnehmung für eigene Kompetenzen und Fähigkeiten der SHTBetroffenen und der Beurteilung ihrer SHT-spezifischen gbzLQ. Im Fall einer bei SHT-Betroffenen häufig vorliegenden gestörten Selbstwahrnehmung im Sinne einer unrealistisch positiven Beurteilung der eigenen Fähigkeiten (Anosognosie) ist eine ebenfalls erhöhte Beurteilung der gbzLQ zu vermuten, die in dieser spezifischen Untergruppe gesondert berücksichtigt werden sollte.

Die hier erbrachten Ergebnisse zeigen ferner, dass unterschiedliche spezifische Verarbeitungsstrategien von den Betroffenen angewendet werden, um die Konsequenzen des erlittenen Traumas zu bewältigen, und jeweils auch in unterschiedlichem Zusammenhang mit der gbzLQ-Beurteilung stehen. 
Selbstwahrnehmung bzw. gestörte Selbstwahrnehmung und die bei der Krankheitsverarbeitung verwendeten Strategien können somit als bedeutende Einflussgrößen im Modell der SHT-spezifischen gbzLQ bestätigt werden und unterstreichen die klinisch-praktische Relevanz der subjektiven Perspektive für die Versorgung und Rehabilitation von SHT-Betroffenen. Gleichzeitig stellen die an diesem speziellen Beispiel gefundenen Zusammenhänge einen Beitrag zur Aufdeckung von systematischen Beziehungen zwischen allgemeinen Aspekten des menschlichen Erlebens und Verhaltens dar. 


\section{LITERATURVERZEICHNIS}

(Die Literaturangaben zu den ausschließlich in den Publikationen zitierten Quellen sind den dort angeführten Literaturverzeichnissen zu entnehmen.)

Anson, K. \& Ponsford, J. (2006). Coping and emotional adjustment following traumatic brain injury. Journal of Head Trauma and Rehabilitation, 21, 248-259.

AWMF, Arbeitsgemeinschaft der Wissenschaftlichen Medizinischen Fachgesellschaft, online, Leitlinien der Deutschen Gesellschaft für Neurochirurgie, AWMF-Leitlinien-Register Nr. 008/001 Entwicklungsstufe: 2e, in Überarbeitung. Zugriff am 26.06.2014 unter http://www.awmf.org/uploads/tx_szleitlinien/008001_S2e_Schaedel-Hirn-trauma_im_Erwachsenenalter_leitlinientext_abgelaufen.pdf

Babinski, J. (1914). Contribution à l'étude des troubles mentaux dans l'hemiplégie organique cérébrale (Anosognosie). Revue Neurologique, 27, 845-847.

Bach, L. J. \& David, A. S. (2006). Self-awareness after acquired and traumatic brain injury. Neuropsychological Rehabilitation,16, 397-414.

Baguley, I. J., Cooper, J. \& Felmingham, K. (2006). Aggressive behaviour following traumatic brain injury. How common is common? Journal of Head Trauma and Rehabilitation, 21, 45-56.

Behan, L. A. \& Agha, A. (2007). Endocrine consequences of adult traumatic brain injury. Hormone Research, (Suppl. 5), 18-21.

Borgaro, S. R. \& Prigatano, G. P. (2003). Modifications of the Patient Competency Rating Scale for use on an acute neurorehabilitation unit: the PCRS-NR. Brain Injury,17, 847-853.

Brüderl, L., Halsig, N. \& Schröder, A. (1988). Historischer Hintergrund, Theorien und Entwicklungstendenzen der Bewältigungsforschung. In L. Brüderl (Hrsg.), Theorien und Methoden der Bewältigungsforschung (S. 25-45). Weinheim: Juventa.

Bullinger, M. (2000). Lebensqualität - Aktueller Stand und neuere Entwicklungen der internationalen Lebensqualitätsforschung. In U. Ravens-Sieberer \& A. Cieza (Hrsg.), Lebensqualität und Gesundheitsökonomie in der Medizin. Konzepte, Methoden, Anwendung (S. 13-24). Landsberg: Ecomed.

Bullinger, M. (2002). Assessing health-related quality of life in medicine. An overview over concepts, methods and applications in international research. Restorative Neurology and Neuroscience, 20, 93-101.

Cantor, J. B., Ashman, T., Gordon, W., Ginsberg, A., Engman, C., Egan, M. et al. (2008). Fatigue after traumatic brain injury and its impact on participation and quality of life. Journal of Head Trauma and Rehabilitation, 23, 41-51.

Carlozzi, N. E., Tulsky, D. S. \& Kisala, P. A. (2011). Traumatic brain injury patient-reported outcome measure: identification of health-related quality-of-life issues relevant to individuals with traumatic brain injury. Archives of Physical Medicine and Rehabilitation, 92 (Suppl. 1), 52-60.

Casper, S. (1991). Zur wissenschaftlichen Fundierung eines Modells der Lebensqualität. In M. Bullinger, M. Ludwig \& N. von Steinbüchel (Hrsg.), Lebensqualität bei kardiovaskulären Erkrankungen. Grundlagen, Meßverfahren und Ergebnisse (S. 35-40). Göttingen: Hogrefe. 
Castriotta, R. J., Wilde, M. C., Lai, J. M., Atanasov, S., Masel, B. E. \& Kuna, S. T. (2007). Prevalence and consequences of sleep disorders in traumatic brain injury. Journal of Clinical Sleep Medicine, 3, 349-356.

Colantonio, A., Ratcliff, G., Chase, S., Kelsey, S., Escobar, M. \& Vernich, L. (2004). Long-term outcomes after moderate to severe traumatic brain injury. Disability and Rehabilitation, 26, 253-261.

Corrigan, J. D. \& Deutschle, J. J. (2008). The presence and impact of traumatic brain injury among clients in treatment for co-occurring mental illness and substance abuse. Brain Injury, 22, 223231.

Dikmen, S. S., Machamer, J. E., Powell, J. M. \& Temkin, N. R. (2003). Outcome 3 to 5 years after moderate to severe traumatic brain injury. Archives of Physical Medicine and Rehabilitation, 84, 1449-1456.

Dirette, D. (2002). The development of awareness and the use of compensatory strategies for cognitive deficits. Brain Injury, 16, 861-871.

Fearnside, M. R. \& Simpson, D. A. (2005). Epidemiology. In P. L. Reilly \& R. Bullock (Eds.), Head Injury. Pathophysiology and Management (pp. 3-26). New York: Arnold.

Fischer, S., Gauggel, S. \& Trexler, L. E. (2004). Awareness of activity limitations, goal setting and rehabilitation outcome in patients with brain injuries. Brain Injury, 18, 547-562.

Fleming, J. M., Strong, J. \& Ashton, R. (1996). Self-awareness of deficits in adults with traumatic brain injury: how best to measure? Brain Injury, 10, 1-15.

Folkman, S. \& Lazarus, R. S. (1980). An analysis of coping in a middle-aged community sample. Journal of Health and Social Behavior, 21, 219-239.

Gauggel, S., Konrad, K. \& Wietasch, A.-K. (1998). Neuropsychologische Rehabilitation. Ein Kompetenz- und Kompensationsprogramm. Weinheim: Psychologie Verlags Union.

Godfrey, H., Knight, R. G. \& Partridge, F. M. (1996). Emotional adjustment following traumatic brain injury: a stress-appraisal-coping formulation. Journal of Head Trauma and Rehabilitation, 11, 29-40.

Goldenberg, G. (1997). Neuropsychologie: Grundlagen, Klinik, Rehabilitation. Stuttgart: Gustav Fischer.

Halligan, P. W. (2006). Awareness and knowing: implications for rehabilitation. Neuropsychological Rehabilitation, 16, 456-473.

Henze, K.-H. (1994). Angst, Abwehr und Bewältigung bei bzw. von Krankheit. In U. Oelke, H.-J. Flor \& G. Ruwe (Hrsg.), Lernen in der Pflege. Gesundsein-Kranksein und Umwelt (S. 50-111). Baunatal: Baunataler Verlag und Software.

Herschbach, P. (2012). Lebensqualität und Lebenszufriedenheit. In E. Brähler \& B. Strauß (Hrsg.), Grundlagen der Medizinischen Psychologie (Enzyklopädie der Psychologie, Themenbereich D: Praxisgebiete, Serie VIII: Medizinische Psychologie, Bd. 1, S. 513-539). Göttingen: Hogrefe.

Holmes, T. H. \& Rahe, R. H. (1967). The social readjustment scale. Journal of Psychosomatic Research, 11, 213-218. 
Jennett, B. (2005). Outcome prediction after severe head injury. In P. L. Reilly \& R. Bullock (Eds.), Head Injury. Pathophysiology and Management (pp. 441-461). New York: Arnold.

Jennett, B. \& Bond, M. (1976). Assessment of outcome after severe brain damage. Lancet, 1 (7905), 480-484.

Jorge, R. E. (2005). Neuropsychiatric consequences of traumatic brain injury: a review of recent findings. Current Opinion in Psychiatry, 18, 289-299.

Jumisko, E., Lexell, J. \& Söderberg, S. (2005). The meaning of living with traumatic brain injury in people with moderate to severe traumatic brain injury. Journal of Neuroscience Nursing, 37, $42-50$

Kashluba, S., Hanks, R. A., Casey, J. E. \& Millis, S. R. (2008). Neuropsychologic and functional outcome after complicated mild traumatic brain injury. Archives of Physical Medicine and Rehabilitation, 89, 904-911.

Kasten, E., Gothe, J. \& Müller, I. (2003). Psychische Störungen nach Hirnschädigung. Psychomed, 15, 214-221.

Keller, I. \& Grömminger, O. (1993). Aufmerksamkeit. In D. Y. von Cramon, N. Mai, W. Ziegler (Hrsg.). Neuropsychologische Diagnostik (S. 65-90). Basel: VCH.

Kendall, E. \& Terry, D. J. (1996). Psychosocial adjustment following closed head injury: a model for understanding individual differences and predicting outcome. Neuropsychological Rehabilitation, 6, 101-132.

Kendel, F. \& Sieverding, M. (2012). Krankheitsverarbeitung - Coping. In E. Brähler \& B. Strauß (Hrsg.), Grundlagen der Medizinischen Psychologie (Enzyklopädie der Psychologie, Themenbereich D: Praxisgebiete, Serie VIII: Medizinische Psychologie, Bd. 1, S. 461-485). Göttingen: Hogrefe.

Kerekjarto, M. von, Schulz, K.-H., Kramer, C., Fittschen, B. \& Schug, S. (1989). Grundlegende Aspekte zum Konzept der Lebensqualität. In R. Verres \& M. Hasenbring (Hrsg.), Jahrbuch der medizinischen Psychologie, Psychosoziale Onkologie (S. 18-29). Berlin: Springer.

Kiraly, M. A. \& Kiraly, S. J. (2007). Traumatic brain injury and delayed sequelae: a review traumatic brain injury and mild traumatic brain injury (concussion) are precursors to lateronset brain disorders, including early-onset dementia. The Scientific World Journal, 7, 17681776.

Koch, U. (2000). Geleitwort. In U. Ravens-Sieberer \& A. Cieza (Hrsg.). Lebensqualität und Gesundheitsökonomie in der Medizin. Konzepte, Methoden, Anwendung (S. 9-10). Landsberg: Ecomed.

Kolb, B. \& Whishaw, I. Q. (1996). Neuropsychologie. Heidelberg: Spektrum Akademischer Verlag.

Koponen, S., Taiminen, T., Portin, R., Himanen, L., Isoniemi, H., Heinonen, H. et al. (2002). Axis I and II psychiatric disorders after traumatic brain injury: a 30-year follow-up study. American Journal of Psychiatry, 159, 1315-1321.

Krpan, M. K., Anderson, N. D. \& Stuss, D. T. (2013). Obstacles to remediating coping following traumatic brain injury. NeuroRehabilitation, 32, 721-728. 
Krpan, M. K., Levine, B., Stuss, D. T. \& Dawson, D. R. (2007). Executive function and coping at oneyear post traumatic brain injury. Journal of Clinical and Experimental Psychology, 29, 36-46.

Lazarus, R. S. (1966). Psychological Stress and the Coping Process. New York: McGraw-Hill.

Lazarus, R. S. \& Folkman, S. (1984). Stress, Appraisal, and Coping. New York: Springer.

Lequerica, A. H., Rapport, L. J., Loeher, K., Axelrod, B. N., Vangel, S. J. \& Hanks, R. A. (2007). Agitation in acquired brain injury: impact on acute rehabilitation therapies. Journal of Head Trauma and Rehabilitation, 22, 177-183.

Lombard, L.A. \& Zafonte, R. D. (2005). Agitation after traumatic brain injury. Considerations and treatment options. American Journal of Physical Medicine and Rehabilitation, 84, 797-812.

Lovasik, D., Kerr, M. E. \& Alexander, S. (2001). Traumatic brain injury research: a review of clinical studies. Critical Care Nursing Quarterly, 23, 24-41.

Lundqvist, A. \& Alinder, J. (2007). Driving after brain injury: self-awareness and coping at the tactical level of control. Brain Injury, 2, 1109-1117.

Maas, A. I. R., Stocchetti, N. \& Bullock, R. (2008). Moderate and severe traumatic brain injury in adults. Lancet Neurology, 7, 728-741.

Maegele, M., Engel, D., Bouillon, B., Lefering, R., Fach, H., Raum, M. et al. (2007). Incidence and outcome of traumatic brain injury in an urban area in western Europe over 10 years. European Surgical Research, 39, 372-379.

Malec, J. F., Brown, A. W., Moessner, A. M., Stump, T. E. \& Monahan, P. (2010). A preliminary model for posttraumatic brain injury depression. Archives of Physical Medicine and Rehabilitation, 91, 1087-1097.

Malec, J. F., Testa, J. A., Rush, B. K., Brown, A. W. \& Moessner, A. M. (2007). Self-assessment of impairment, impaired self-awareness, and depression after traumatic brain injury. Journal of Head Trauma and Rehabilitation, 22, 156-166.

Malia, K., Powell, G. \& Torode, S. (1995). Coping and psychosocial function after brain injury. Brain Injury, 9, 607-618.

Markova, I. S. \& Berrios, G. E. (2006). Approaches to the assessment of awareness: conceptual issues. Neuropsychological Rehabilitation, 16, 439-455.

Maskell, F., Chiarelli, P. \& Isles, R. (2007). Dizziness after traumatic brain injury: results from an interview study. Brain Injury, 2, 741-752.

Meadows, K. A. (2011). Patient-reported outcome measures: an overview. British Journal of Community Nursing, 16, 146-151.

Medley, A. R., Powell, T., Worthington, A., Chodan, G. \& Jones, C. (2010). Brain injury beliefs, selfawareness, and coping: a preliminary cluster analytic study based within the self-regulatory model. Neuropsychological Rehabilitation, 20, 899-921.

Mesulam, M. M. (1985). Principles of Behavioral Neurology. Philadelphia: Davis. 
Muthny, F. A. (1989a). Erfahrungen mit dem Freiburger Fragebogen zur Krankheitsverarbeitung (FKV). In R. Verres \& M. Hasenbring (Hrsg.), Jahrbuch der medizinischen Psychologie (Psychosoziale Onkologie, Bd. 3, S. 86-101). Berlin: Springer.

Muthny, F. A. (1989b). Freiburger Fragebogen zur Krankheitsverarbeitung. Manual. Weinheim: Beltz Test.

Muthny, F. A. (1990). Zur Spezifität der Krankheitsverarbeitung. In F. A. Muthny (Hrsg.), Krankheitsverarbeitung. Hintergrundtheorien, klinische Erfassung und empirische Ergebnisse. Berlin: Springer.

Navratil, O., Smrcka, M. \& Hannak, P. (2006). The outcome, working ability and psychic changes after traumatic brain injury. Bratislava Medical Journal-Bratislavské Lekárske Listy, 107, $110-112$.

Parcell, D. L., Ponsford, J. L., Redman, J. R. \& Rajaratnam, S. (2008). Poor sleep quality and changes in objectively recorded sleep after traumatic brain injury: a preliminary study. Archives of Physical Medicine and Rehabilitation, 89, 843-850.

Poeck, K. (1997). Anosognosie. In: W. Hartje \& K. Poeck (Hrsg.). Klinische Neuropsychologie. Stuttgart: Georg Thieme.

Ponsford, J. (2013). Factors contributing to outcome following traumatic brain injury. NeuroRehabilitation, 32, 803-815.

Ponsford, J. L., Downing, M. G., Olver, J., Ponsford, M., Acher, R., Carty, M. et al. (2014). Longitudinal follow-up of patients with traumatic brain injury: outcome at two, five, and ten years post-injury. Journal of Neurotrauma, 31, 64-77.

Prigatano, G. P. (1991). Disturbances of self-awareness of deficit after traumatic brain injury. In G. Prigatano \& D. L. Schacter (Eds.), Awareness of Deficit After Brain Injury. Clinical and Theoretical Issues (pp. 111-126). Oxford: Oxford University Press.

Prigatano, G. P. (1992). Neuropsychological rehabilitation and the problem of altered self-awareness. In N. von Steinbüchel, D. Y. von Cramon \& E. Pöppel (Eds.), Neuropsychological Rehabilitation. Berlin: Springer.

Prigatano, G. P. (1996). Anosognosia. In J. G. Beaumont, P. M. Kenealy \& M. J. C. Rogers (Eds.), The Blackwell Dictionary of Neuropsychology (pp. 80-84). Cambridge, Mass.: Blackwell.

Prigatano, G. P. (2004). Neuropsychologische Rehabilitation. (S. Fischer \& J. Küst, Übers.). Heidelberg: Springer. (Original erschienen 1999: Principles of Neuropsychological Rehabilitation)

Prigatano, G. P. (2005). Disturbances of self-awareness and rehabilitation of patients with traumatic brain injury. A 20-year perspective. Journal of Head Trauma and Rehabilitation, 29, 19-29.

Prigatano, G. P., Fordyce, D. J., Zeiner, H. K., Roueche, J. R., Pepping, M. \& Wood, B. C. (1986). Neuropsychological rehabilitation after brain injury. Baltimore and London: The John Hopkins University Press.

Prigatano, G. P. \& Schacter, D. L. (1991). Introduction. In G. Prigatano \& D. L. Schacter (Eds.), Awareness of Deficit After Brain Injury. Clinical and Theoretical Issues (pp. 4-16). Oxford: Oxford University Press. 
Rao, V., Spiro, J., Vaishnavi, S., Rastogi, P., Mielke, M., Noll, K. et al. (2008). Prevalence and types of sleep disturbances acutely after traumatic brain injury. Brain Injury, 22, 381-386.

Ravens-Sieberer, U. \& Cieza, A. (2000). Lebensqualitätsforschung in Deutschland - Forschungsstand, Methoden, Anwendungsbeispiele und Implikationen. In U. Ravens-Sieberer \& A. Cieza (Hrsg.), Lebensqualität und Gesundheitsökonomie in der Medizin. Konzepte, Methoden, Anwendung (S. 25-49). Landsberg: Ecomed.

Rickels, E. (2006a). Epidemiologie. In E. Rickels, K. von Wild, P. Wenzlaff \& W. J. Bock (Hrsg.), Schädel-Hirn-Verletzung. Epidemiologie und Versorgung. Ergebnisse einer prospektiven Studie (S. 107-114). München: Zuckschwerdt.

Rickels, E. (2006b). Hochrechnung der wesentlichen Fakten auf die Bundesrepublik. In E. Rickels, K. von Wild, P. Wenzlaff \& W. J. Bock (Hrsg.), Schädel-Hirn-Verletzung. Epidemiologie und Versorgung. Ergebnisse einer prospektiven Studie (S. 217-218). München: Zuckschwerdt.

Rüger, U., Blomert, A. F. \& Förster, W. (1990). Coping. Theoretische Konzepte, Forschungsansätze, Messinstrumente zur Krankheitsbewältigung. Göttingen: Vandenhoeck \& Ruprecht.

Sasse, N., Gibbons, H., Wilson, L., Martinez-Olivera, R., Schmidt, H., Hasselhorn, M., Wild, K. von \& Steinbüchel, N. von (2013). Self-awareness and health-related quality of life after traumatic brain injury. Journal of Head Trauma and Rehabilitation, 28, 464-472 (published ahead-ofprint August 29, 2012).

Sasse, N., Gibbons, H., Wilson, L., Martinez, R., Sehmisch, S., Wild, K. von et al. (2014). Coping strategies in individuals after traumatic brain injury: Associations with health-related quality of life. Disability and Rehabilitation, March 3 early online: 1-9, DOI: 10.3109/09638288.2014.893029.

Schönberger, M., Humle, F. \& Teasdale, T. W. (2006a). Subjective outcome of brain injury rehabilitation in relation to the therapeutic working alliance, client compliance and awareness. Brain Injury, 20, 1271-1282.

Schönberger, M., Humle, F., Zeeman, P. \& Teasdale, T. W. (2006b). Patient compliance in brain injury rehabilitation in relation to awareness and cognitive and physical improvement. Neuropsychological Rehabilitation, 16, 561-578.

Schönberger, M., Ponsford, J., Gould, K. R. \& Johnston, L. (2011). The temporal relationship between depression, anxiety, and functional status after traumatic brain injury: a cross-lagged analysis. Journal of the International Neuropsychological Society, 17, 781-787.

Schumacher, J., Klaiberg, A. \& Brähler, E. (2003). Diagnostik von Lebensqualität und Wohlbefinden - Eine Einführung. In J. Schumacher, A. Klaiberg \& E. Brähler (Hrsg.), Diagnostische Verfahren zu Lebensqualität und Wohlbefinden. Diagnostik für Klinik und Praxis (Bd. 2, 9-23). Göttingen: Hogrefe.

Selye, H. (1976). The Stress of Life. New York: McGraw-Hill.

Shotton, L., Simpson, J. \& Smith, M. (2007). The experience of appraisal, coping and adaptive psychosocial adjustment following traumatic brain injury: a qualitative investigation. Brain Injury, 21, 857-869.

Skandsen, T., Finnanger, T. G., Andersson, S., Lydersen, S., Brunner, J. F. \& Vik, A. (2010). Cognitive impairment 3 months after moderate and severe traumatic brain injury: a 
prospective follow-up study. Archives of Physical Medicine and Rehabilitation, 91, 19041913.

Steinbüchel, N. von (1995). Erfassung von mentalen Funktionen und gesundheitsbezogener Lebensqualität. Unveröffentlichte Habilitationsschrift. Universität Innsbruck.

Steinbüchel, N. von, Petersen, C., Bullinger, M. \& the QOLIBRI Group (2005a). Assessment of health-related quality of life in persons after traumatic brain injury - development of the QOLIBRI, a specific measure. Acta Neurochirurgica, 93 (Suppl.), 43-49.

Steinbüchel, N. von, Richter, S., Morawetz, C. \& Riemsma, R. (2005b). Assessment of subjective health and health-related quality of life in persons with acquired or degenerative brain injury. Current Opinion in Neurology, 18, 681-691.

Steinbüchel, N. von, Sasse, N., Otto, C., Gibbons, H., Mullins, R., Behr, R. et al. (2014). Validation of the German language version of the Quality of Life after Brain Injury (QOLIBRI) scale. Manuscript submitted for publication.

Steinbüchel, N. von, Wilson, L., Gibbons, H., Hawthorne, G., Höfer, S., Schmidt, S. et al. (2010a). Quality of Life after Brain Injury (QOLIBRI): Scale development and metric properties. Journal of Neurotrauma, 27, 1167-1185.

Steinbüchel, N. von, Wilson, L., Gibbons, H., Hawthorne, G., Höfer, S., Schmidt, S. et al. (2010b). Quality of Life after Brain Injury (QOLIBRI): Scale validity and correlates of quality of life. Journal of Neurotrauma, 27, 1157-1165.

Steinbüchel, N. von, Wilson, L., Gibbons, H., Mühlan, H., Schmidt, H., Schmidt, S. et al. (2012). QOLIBRI overall scale: A brief index of health-related quality of life after traumatic brain injury. Journal of Neurology, Neurosurgery, and Psychiatry, 83, 1041-1047.

Steinbüchel, N. von, Woerner, W., Sasse, N., Höfer, S., Truelle, J.-L., Wild, K. von et al. (2008). Lebensqualität nach Schädelhirntrauma - erste Ergebnisse einer internationalen Studie. In A. Ebert, W. Fries \& L. Ludwig (Hrsg.), Rehabilitation und Nachsorge nach Schädelhirnverletzung: Möglichkeit und Wirklichkeit 2008 (ZNS Reihe Zentrales Nervensystem, Bd. 2, S. 1522). Bad Honnef: Hippocampus.

Tagliaferri, F., Compagnone, C., Korsic, M., Servadei, F. \& Kraus, J. (2006). A systematic review of brain injury epidemiology in Europe. Acta Neurochirurgica, 148, 255-268.

Tateno, A., Jorge, R. E. \& Robinson, R. G. (2003). Clinical correlates of aggressive behaviour after traumatic brain injury. Journal of Neuropsychiatry and Clinical Neurosciences, 15, 155-160.

Teasdale, G. \& Jennett, B. (1974). Assessment of coma and impaired consciousness. A practical scale. Lancet, 2 (7872), 81-84.

Tomberg, T., Toomela, A., Ennok, M. \& Tikk, A. (2007). Changes in coping strategies, social support, optimism and health-related quality of life following traumatic brain injury: a longitudinal study. Brain Injury, 21, 479-488.

Tomberg, T., Toomela, A., Pulver, A. \& Tikk, A. (2005). Coping strategies, social support, life orientation and health-related quality of life following traumatic brain injury. Brain Injury, 19, 1181-1190.

Truelle, J.-L., Fayol, P., Montreuil, M. \& Chevignard, M. (2010). Community integration after severe traumatic brain injury in adults. Current Opinion in Neurology, 23, 688-694. 
Wallace, C. A. \& Bogner, J. (2000). Awareness of deficits: emotional implications for persons with brain injury and their significant others. Brain Injury, 14, 549-562.

Ware, J. E. \& Sherbourne, C. D. (1992). The MOS 36-Item Short-Form Health Status Survey (SF-36): 1. Conceptual framework and item selection. Medical Care, 30, 473-483.

Warriner, E. M. \& Velikonja, D. (2006). Psychiatric disturbances after traumatic brain injury: neurobehavioral and personality changes. Current Psychiatry Reports, 8, 73-80.

Weis, J. (2008). Krankheitsverarbeitung und Lebensqualität. In R. Bäumer \& A. Maiwald (Hrsg.), Onkologische Pflege (S. 332-339). Stuttgart: Georg Thieme.

Whitnall, L., McMillan, T. M., Murray, G. D. \& Teasdale, G. M. (2006). Disability in young people and adults after head injury. 5-7 year follow up of a prospective cohort study. Journal of Neurology, Neurosurgery and Psychiatry, 77, 640-645.

WHO - World Health Organization (1948). Preamble to the Constitution of the World Health Organization as adopted by the International Health Conference, New York, 19-22 June, 1946; signed on 22 July 1946 by the representatives of 61 States (Official Records of the World Health Organization, no. 2, p. 100) and entered into force on 7 April 1948.

Wilson, J. T. L., Pettigrew, L. E. L. \& Teasdale, G. M. (1998). Structured interviews for the Glasgow Outcome Scale and the Extended Glasgow Outcome Scale: guidelines for their use. Journal of Neurotrauma, 15, 573-585.

Wise, K., Ownsworth, T. \& Fleming, J. (2005). Convergent validity of self-awareness measures and their association with employment outcome in adults following acquired brain injury. Brain Injury, 19, 765-775.

Wolters, G., Stapert, S., Brands, I. \& Heugten, C. van (2010). Coping styles in relation to cognitive rehabilitation and quality of life after brain injury. Neuropsychological Rehabilitation, 20, 587-600.

Wood, R. L. (2013a). Neurobehavioural disorders after traumatic brain injury: assessment, treatment and outcome. NeuroRehabilitation, 32, 697-698.

Wood, R. L. (2013b). Recognising and assessing neurobehavioural disability after traumatic brain injury. NeuroRehabilitation, 32, 699-706.

Wood, R. L., Liossi, C. \& Wood, L. (2005). The impact of head injury neurobehavioural sequelae on personal relationships: preliminary findings. Brain Injury,19, 845-851. 